Supporting Information

\title{
The Substitution Effect of the Trifluoromethyl Group on the Bioactivity in Medicinal Chemistry: Statistical Analysis and Energy Calculations
}

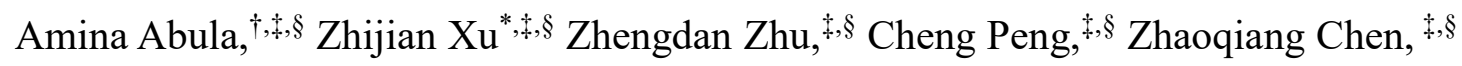

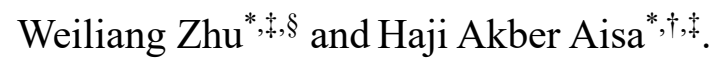

${ }^{\dagger}$ The Key Laboratory of Plant Resources and Chemistry in Arid Regions and Key Laboratory of Xinjiang Indigenous Medicinal Plants Resource Utilization, Xinjiang Technical Institute of Physics and Chemistry, Chinese Academy of Sciences, South Beijing Road 40-1, Urumqi, Xinjiang 830011, People’s Republic of China.

tUniversity of Chinese Academy of Sciences, No. 19A Yuquan Road, Beijing 100049, People's Republic of China.

${ }^{\S}$ CAS Key Laboratory of Receptor Research; Drug Discovery and Design Center, Shanghai Institute of Materia Medica, Chinese Academy of Sciences, 555 Zuchongzhi Road, Shanghai 201203, China.

${ }^{*}$ To whom correspondence should be addressed. Phone: +86-21-50806600-1304 (Z.X.), +86-21-50805020 (W.Z.), +86-991-3835679 (H.A.), E-mail: zjxu@simm.ac.cn (Z.X.),wlzhu@simm.ac.cn (W.Z.), haji@ms.xjb.ac.cn (H.A.). 


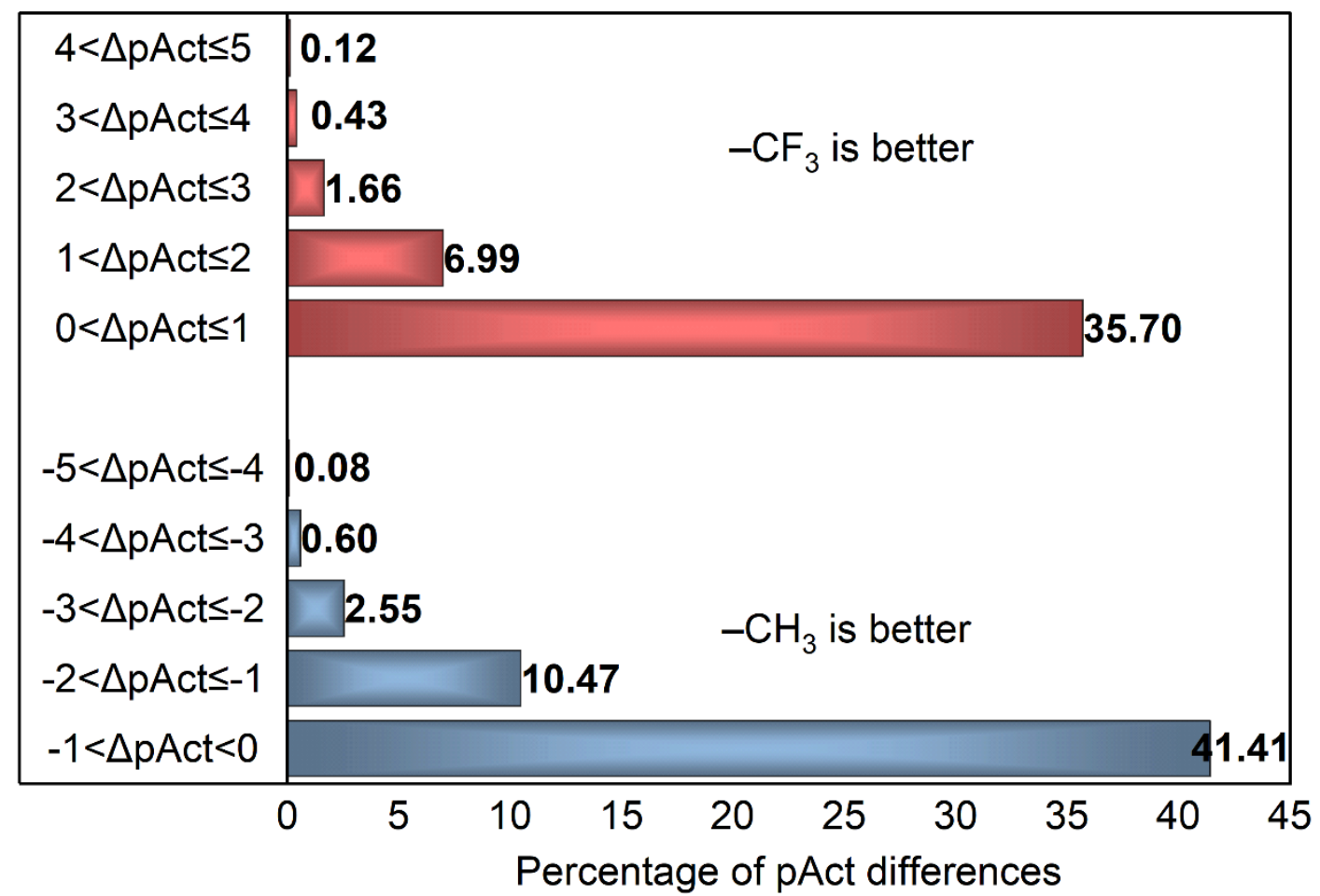

Fig. S1 Distribution of pAct differences in compounds pairs containing $-\mathrm{CF}_{3}$ or $-\mathrm{CH}_{3}$. $\Delta \mathrm{pAct}=\mathrm{pAct}\left(-\mathrm{CF}_{3}\right)-\mathrm{pAct}\left(-\mathrm{CH}_{3}\right)$.

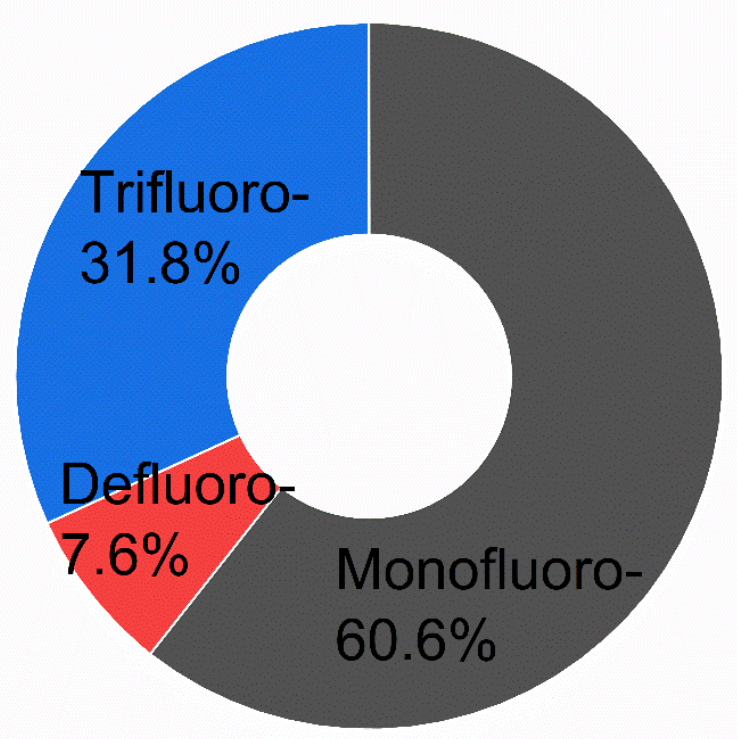

Fig. S2 Distribution of fluoro-containing structures under active development reported in Integrity database (retrieve date: 2020-06-16). 
(a)

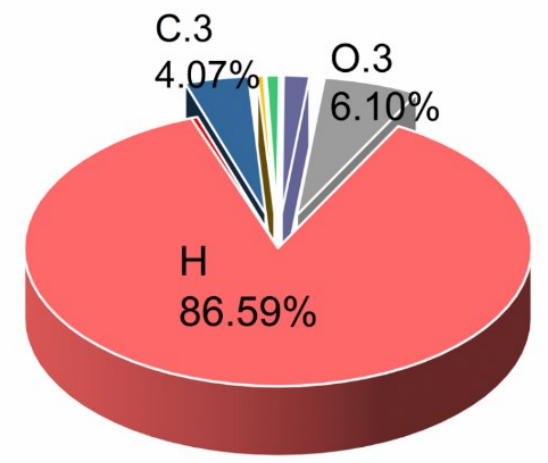

(b)

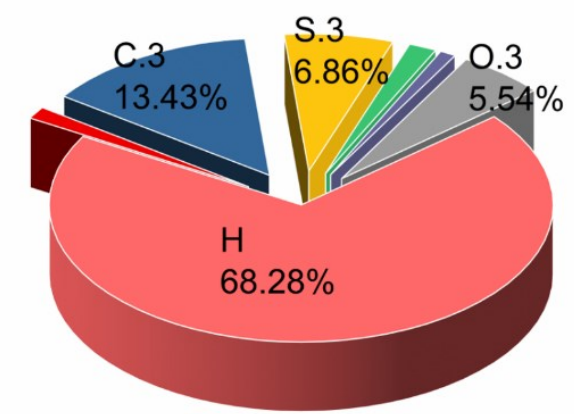

Fig. S3 Distribution of atom types in residues with $\mathrm{RP}>1$ at $d \leq 3 \AA$. Hydrogen (pink), C.3 (blue), O.3 (gray) S.3 (yellow), and O.2 (purple), N.3 (green), C.2 (red). (a) Atomtypes in residues (Ser, His, Trp, Arg, Ala, Tyr, Leu, Met, Phe) interacting with $-\mathrm{CF}_{3}$. (b) Atom-types in residues (Tyr, Trp, Phe, Ile, Cys, Met, Leu) interacting with $-\mathrm{CH}_{3}$. 
(a)

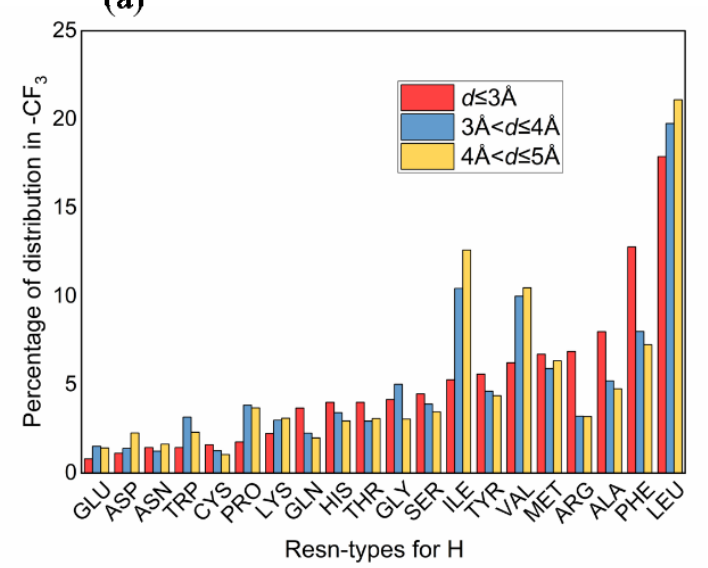

(c)

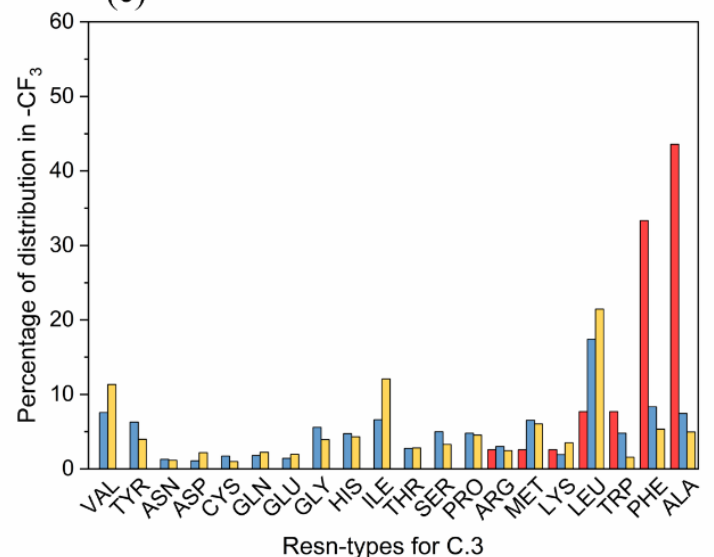

(e)

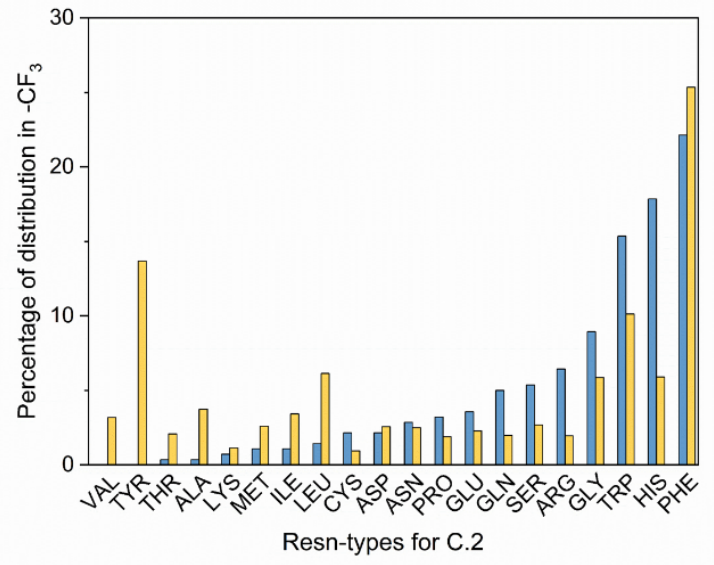

(b)

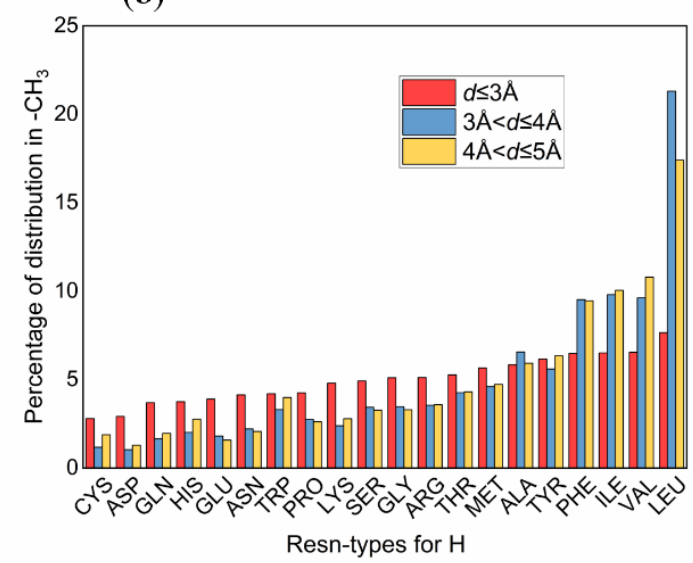

(d)

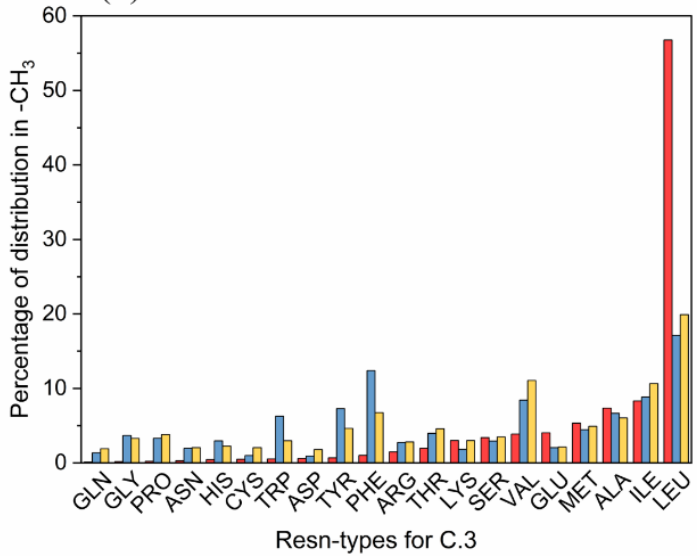

(f)

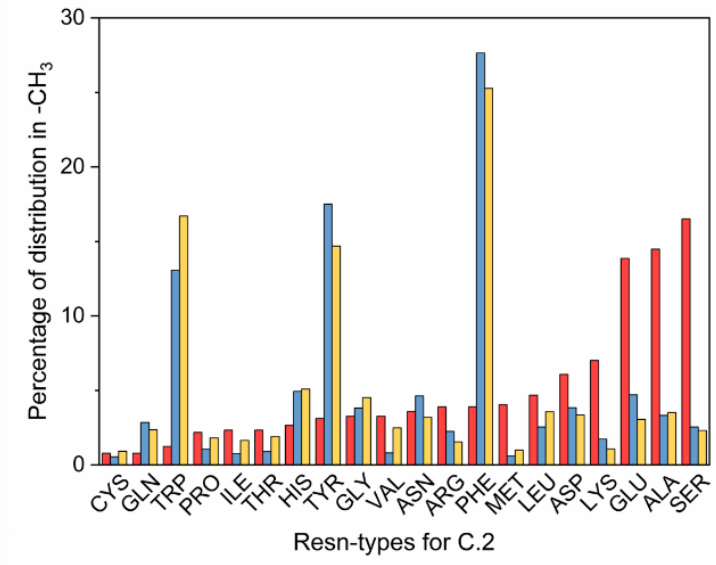


(g)

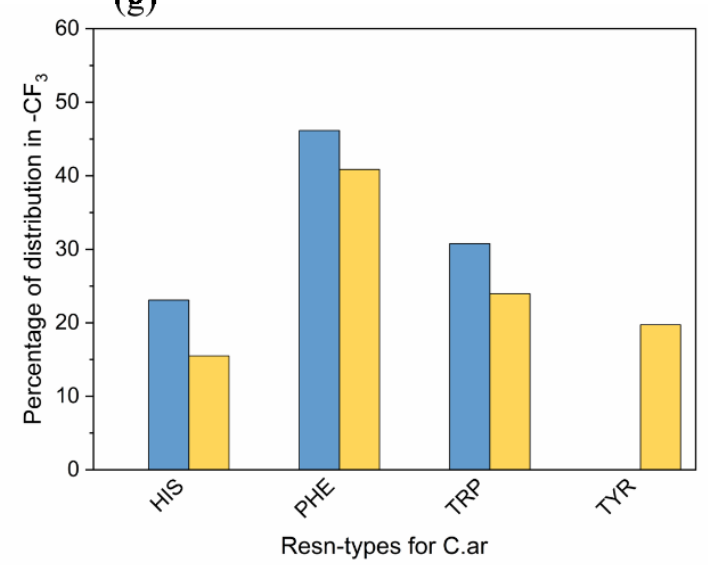

(i)

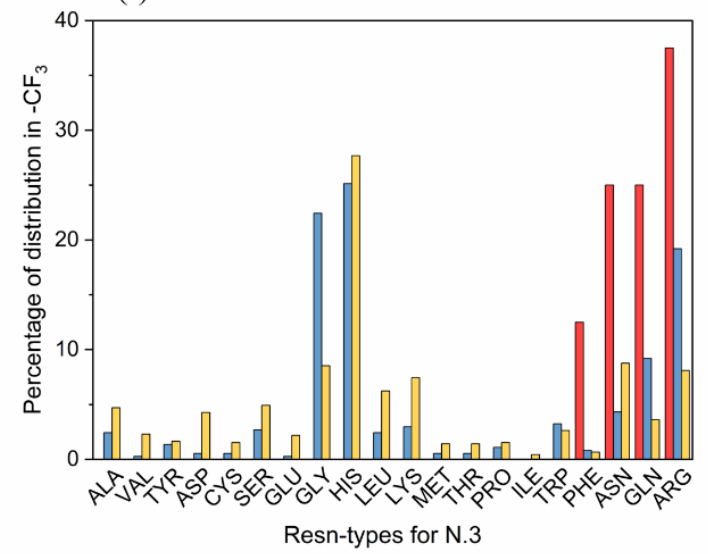

(k)

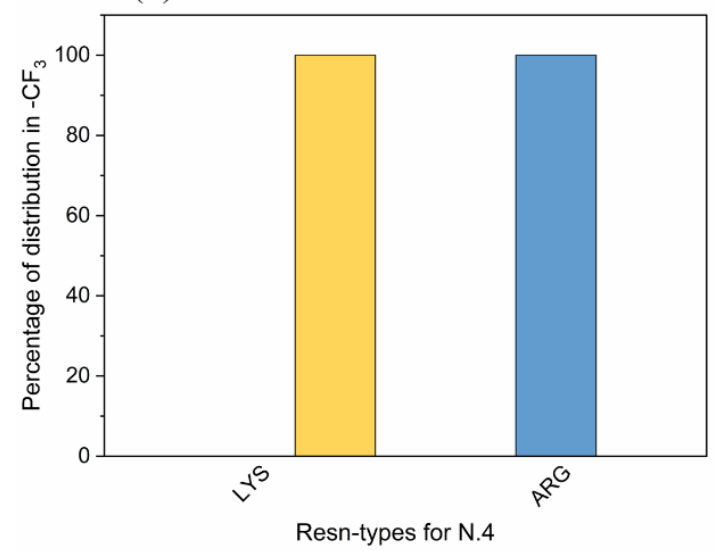

(h)

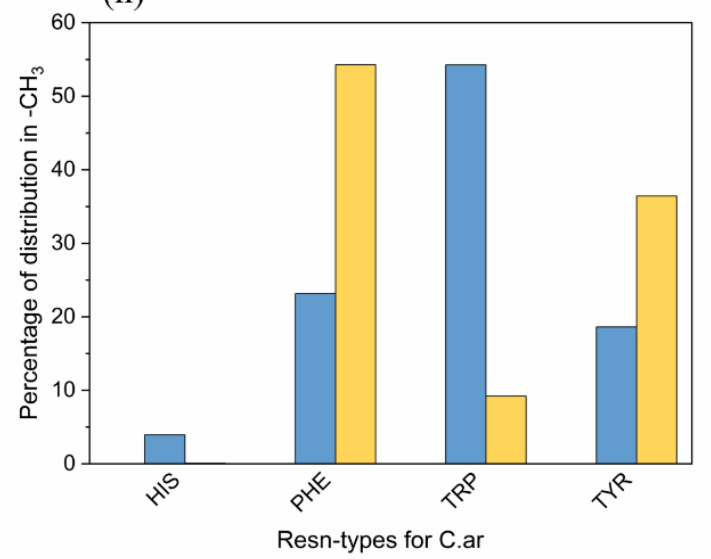

(j)

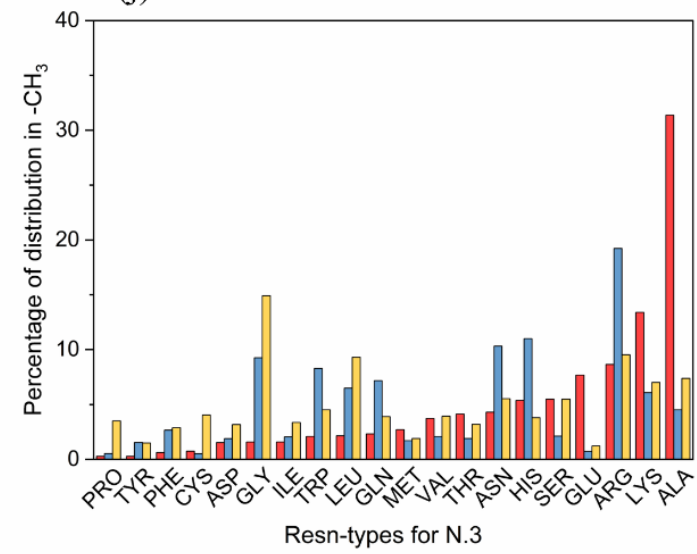

(1)

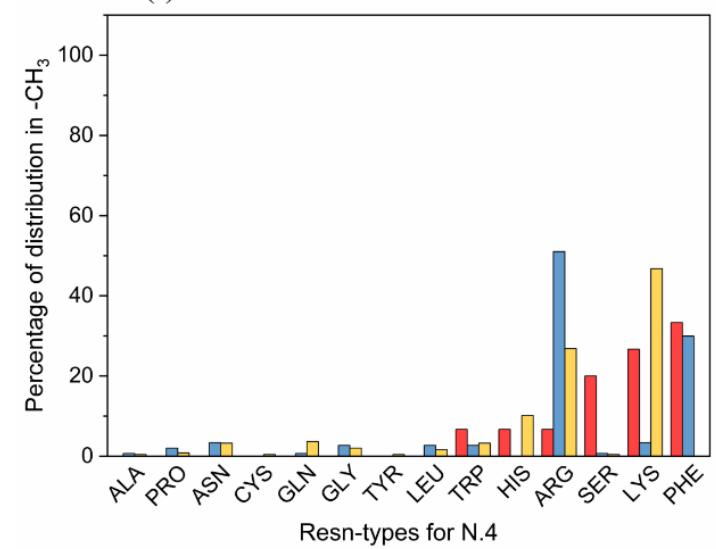


(m)

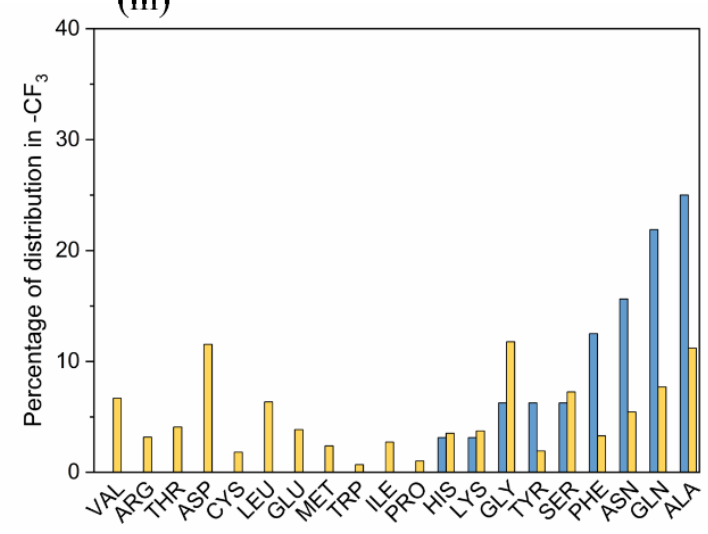

Resn-types for N.am

(o)

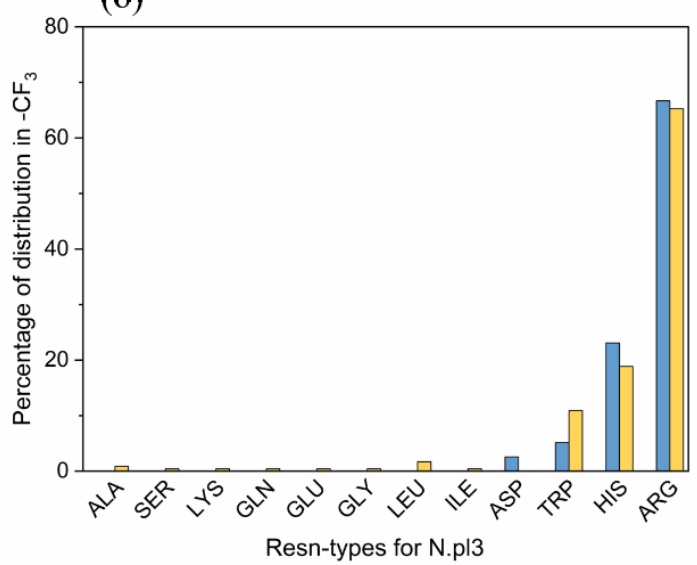

(q)

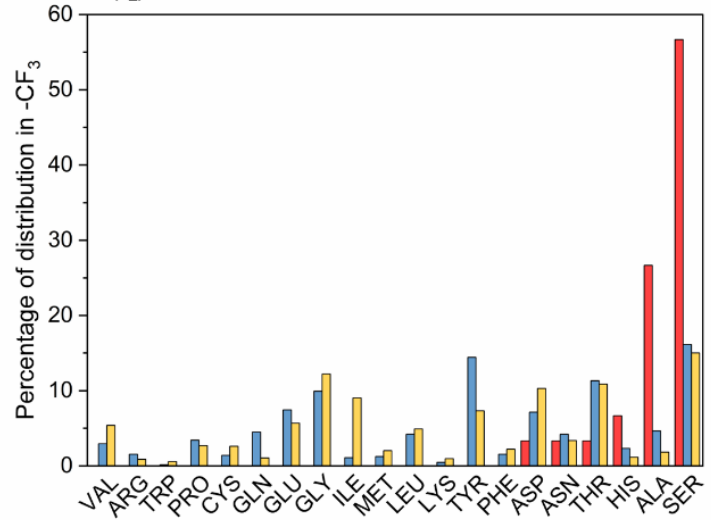

Resn-types for 0.3
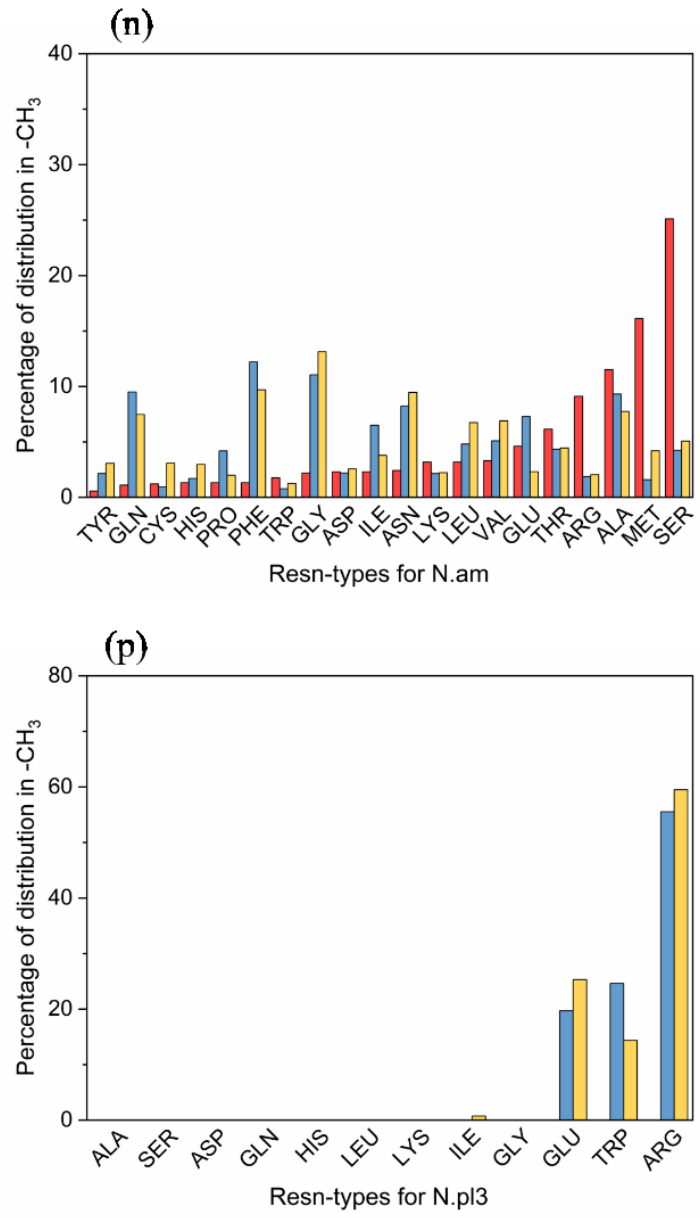

(r)

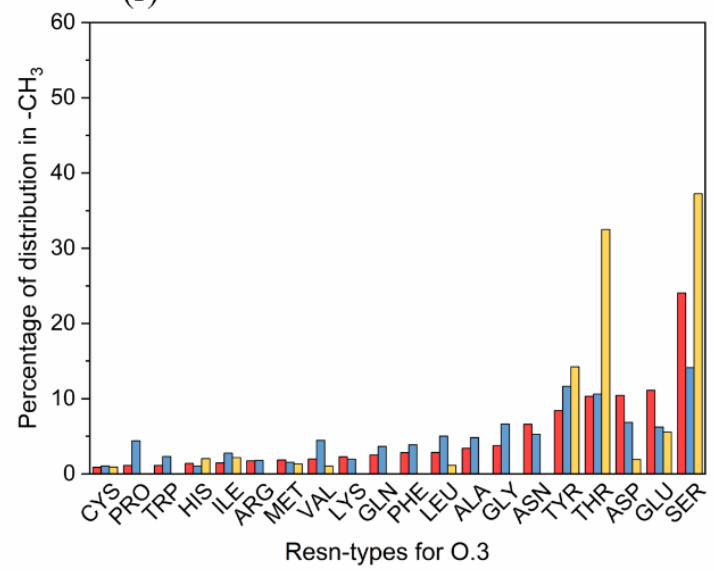


(s)

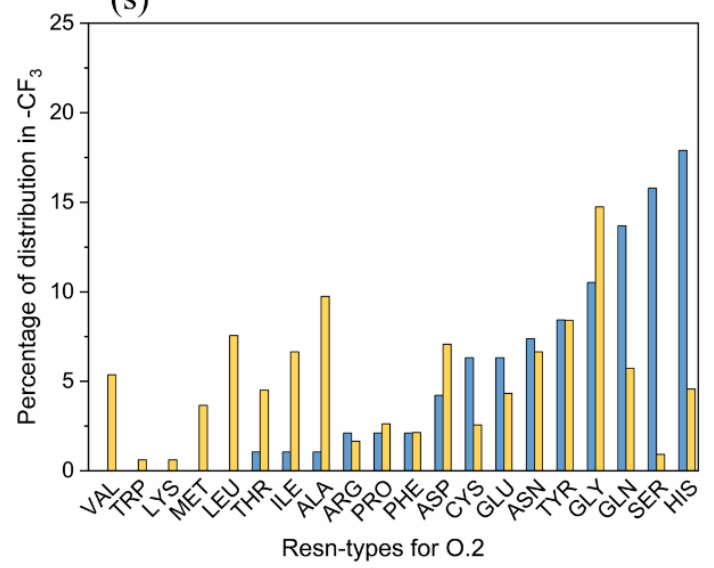

(u)

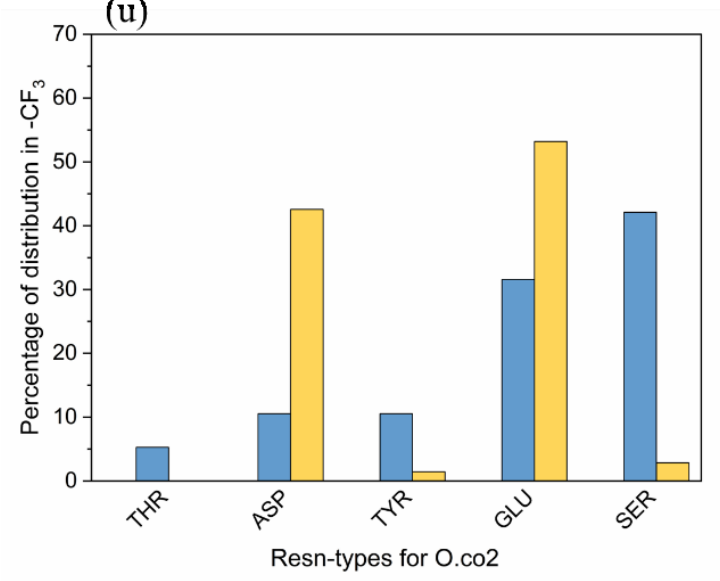

(w)

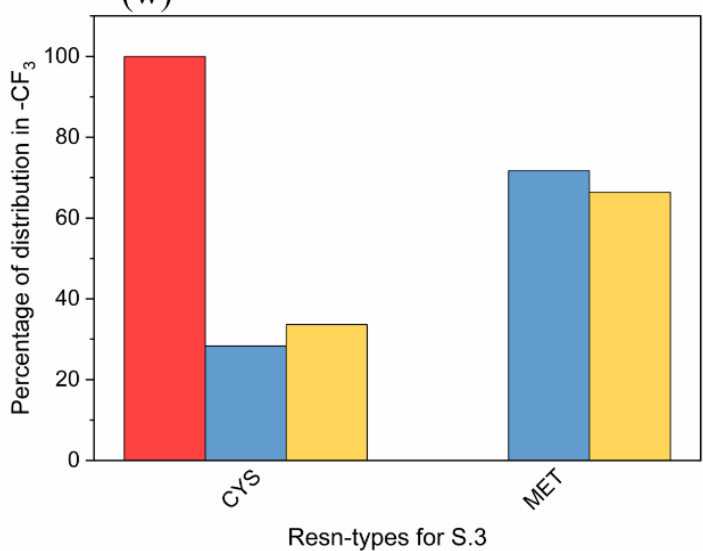

( $)$
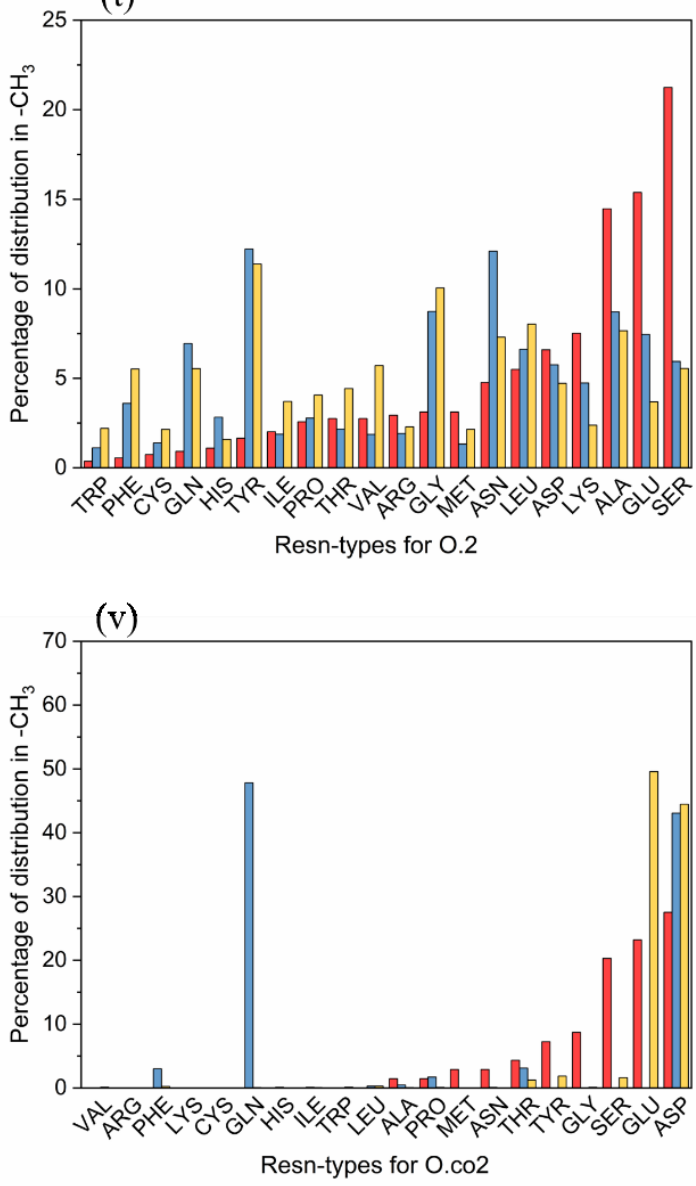

(x)

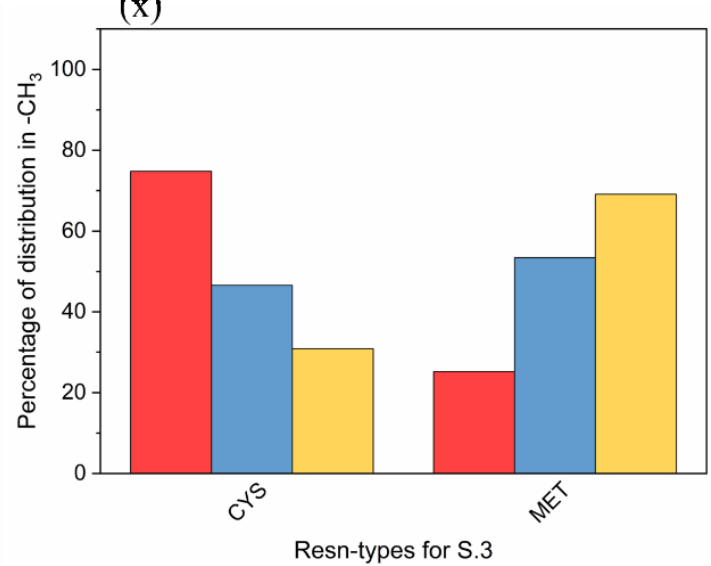

Fig. S4 Percentage of different residues corresponding to 12 atom types (a to $\mathrm{x}$ ) which interact with $-\mathrm{CH}_{3} /-\mathrm{CF}_{3}$ with $d \leq 3 \AA$ (red), $3 \AA<d \leq 4 \AA$ (blue), and $4 \AA<d \leq 5 \AA$ (yellow). 
(a)

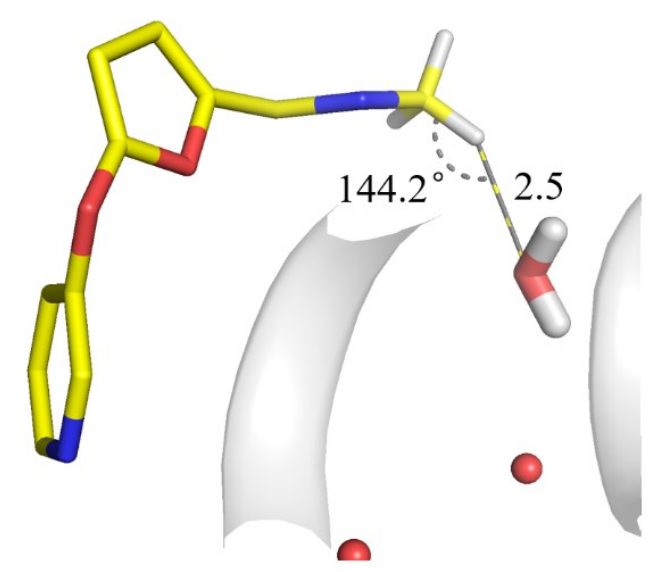

(c)

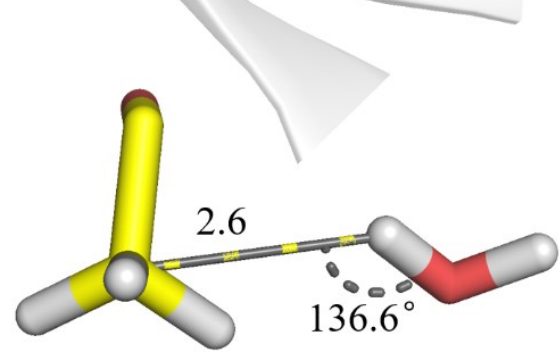

(b)

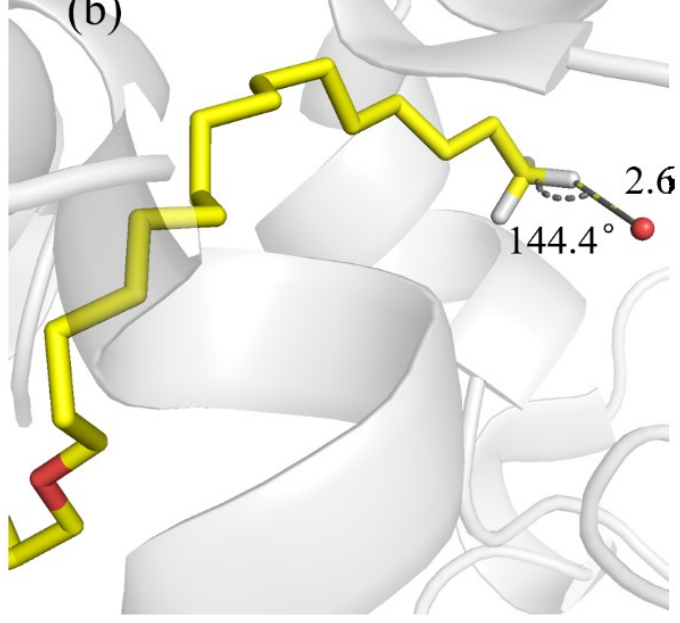

(d)

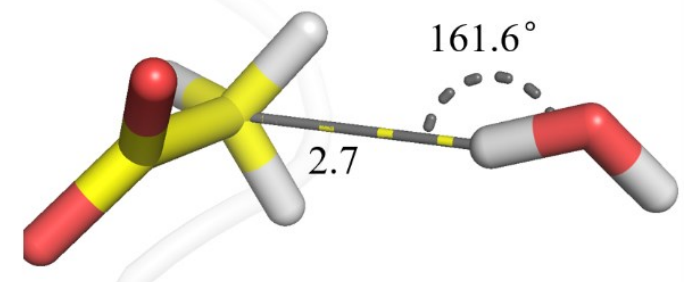

Fig. S5 $\mathrm{C}-\mathrm{H} \cdots \mathrm{O}$ and $\mathrm{O}-\mathrm{H} \cdots \mathrm{C}$ hydrogen bonds between the methyl group and water

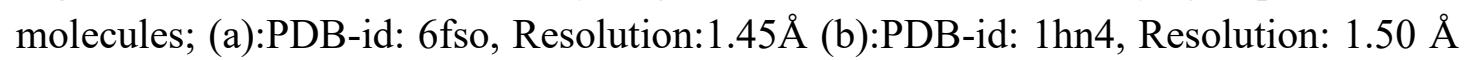

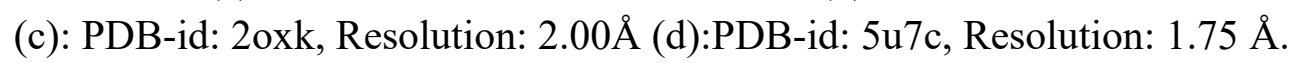




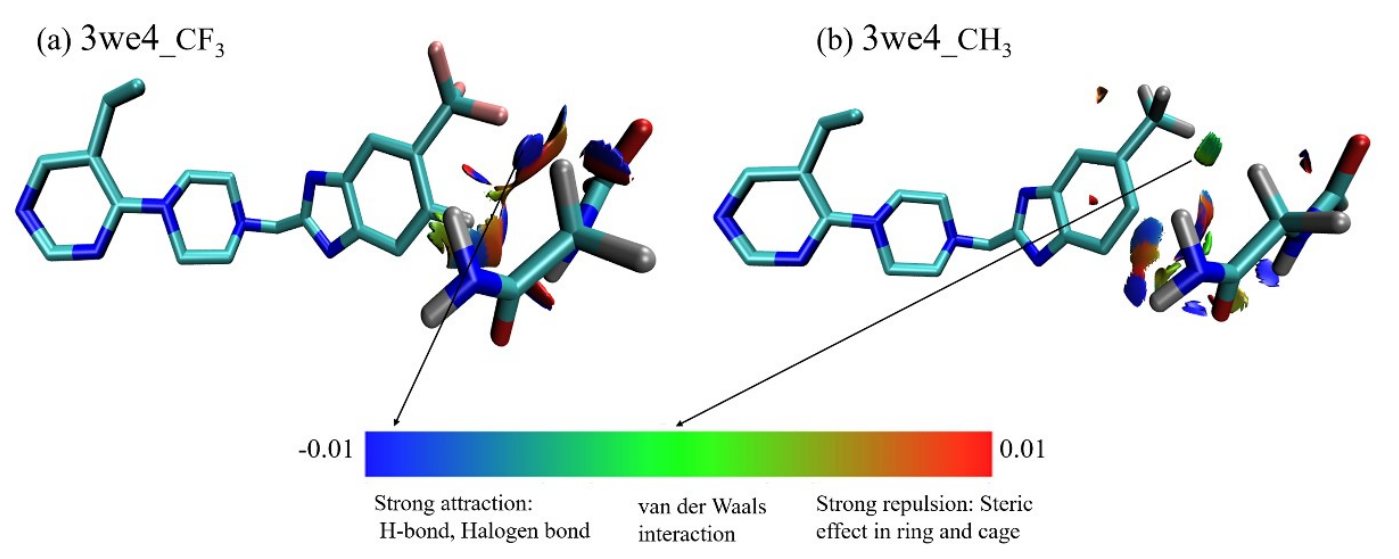

(c) $3 \mathrm{we} 4 \mathrm{CF}_{3}$

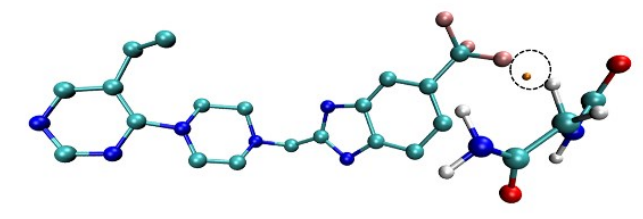

(d) $3 \mathrm{we}_{-} \mathrm{CH}_{3}$

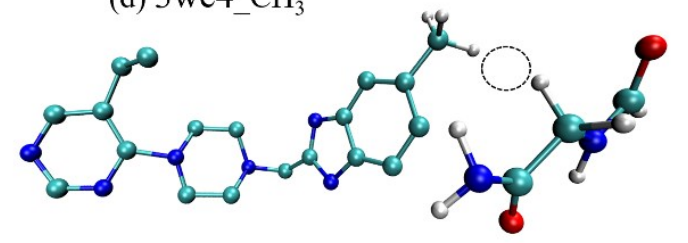

Fig. S6 Non-covalent interactions index (NCI) analysis (a, b) and atoms-in-molecules (AIM) analysis (c, d) for a representative structure (PDB-id: 3 we4) by Multiwfn3. $4^{1}$ $\mathrm{F} \cdots \mathrm{H}\left(-\mathrm{CF}_{3} \cdots \mathrm{H}\right)$ is an attractive interaction (a) while the corresponding $\mathrm{H} \cdots \mathrm{H}(-$ $\mathrm{CH}_{3} \cdots \mathrm{H}$ ) is a very weak van der Waals interaction (b). There is a bond critical point (BCP) between $\mathrm{F} \cdots \mathrm{H}(\mathrm{c})$, implying that an obvious attraction is formed between $\mathrm{F} \cdots \mathrm{H}$, while there is no BCP between $\mathrm{H} \cdots \mathrm{H}(\mathrm{d})$.

Table S1. MOL2 atom types used in this study.

\begin{tabular}{cccc}
\hline Code & Definition & Code & Definition \\
\hline C.3 & carbon $\mathrm{sp}^{3}$ & $\mathrm{O} .3$ & oxygen sp3 \\
C.2 & carbon $\mathrm{sp}^{2}$ & $\mathrm{O} .2$ & $\begin{array}{c}\text { oxygen sp2 } \\
\text { Oxygen in carboxylate } \\
\text { and phosphate groups }\end{array}$ \\
C.ar & carbon aromatic & O.co2 & Hydrogen \\
N.3 & $\begin{array}{c}\text { nitrogen sp } \\
\text { nitrogen sp } \text { spositively }^{3}\end{array}$ & $\mathrm{H}$ & S.3 \\
N.4 & $\begin{array}{c}\text { nitrogen amide } \\
\text { N.am }\end{array}$ & & \\
N.pl3 & nitrogen trigonal planar & & \\
\hline
\end{tabular}


Table S2. The relative residue propensity (RP) for each amino acid in all the proteinligand complexes containing $-\mathrm{CF}_{3}$ group.

\begin{tabular}{|c|c|c|c|c|c|c|c|}
\hline \multicolumn{2}{|c|}{$\begin{array}{c}\text { Intrinsic } \\
\text { Proportion (IP) }\end{array}$} & \multicolumn{3}{|c|}{ Occurrence Proportion (OP) } & \multicolumn{3}{|c|}{ Relative Residues Preferences (RP) } \\
\hline & Freq & $d<3 \AA$ & $3 \AA \leq d<4 \AA$ & $4 \AA \leq d<5 \AA$ & $d<3 \AA$ & $3 \AA \leq d<4 \AA$ & $4 \AA \leq d<5 \AA$ \\
\hline Ala & 7.47 & 10.52 & 5.20 & 4.78 & 1.41 & 0.70 & 0.64 \\
\hline Cys & 2.37 & 1.54 & 1.42 & 1.41 & 0.65 & 0.60 & 0.60 \\
\hline Asp & 5.64 & 1.12 & 1.60 & 2.93 & 0.20 & 0.28 & 0.52 \\
\hline Glu & 7.07 & 0.70 & 1.79 & 2.02 & 0.10 & 0.25 & 0.29 \\
\hline Phe & 3.99 & 13.46 & 7.77 & 8.28 & 3.37 & 1.94 & 2.07 \\
\hline Gly & 6.71 & 3.65 & 5.82 & 4.47 & 0.54 & 0.87 & 0.67 \\
\hline His & 3.00 & 3.79 & 4.49 & 4.81 & 1.26 & 1.50 & 1.60 \\
\hline Ile & 5.99 & 4.63 & 9.06 & 10.13 & 0.77 & 1.51 & 1.69 \\
\hline Lys & 5.65 & 2.10 & 2.68 & 2.86 & 0.37 & 0.47 & 0.51 \\
\hline Leu & 9.15 & 16.13 & 17.76 & 17.21 & 1.76 & 1.94 & 1.88 \\
\hline Met & 2.50 & 6.03 & 5.68 & 5.98 & 2.41 & 2.27 & 2.39 \\
\hline Asn & 4.30 & 1.68 & 1.52 & 1.97 & 0.39 & 0.35 & 0.46 \\
\hline Pro & 4.62 & 1.54 & 3.76 & 3.36 & 0.33 & 0.82 & 0.73 \\
\hline Gln & 4.00 & 3.51 & 2.61 & 2.23 & 0.88 & 0.65 & 0.56 \\
\hline Arg & 4.81 & 6.59 & 3.81 & 3.39 & 1.37 & 0.79 & 0.70 \\
\hline Ser & 6.53 & 7.29 & 4.51 & 3.71 & 1.12 & 0.69 & 0.57 \\
\hline Thr & 5.06 & 3.65 & 3.09 & 3.01 & 0.72 & 0.61 & 0.59 \\
\hline Val & 6.47 & 5.47 & 8.85 & 8.86 & 0.85 & 1.37 & 1.37 \\
\hline Trp & 1.32 & 1.68 & 3.43 & 3.18 & 1.27 & 2.60 & 2.41 \\
\hline Tyr & 3.35 & 4.91 & 5.15 & 5.43 & 1.47 & 1.54 & 1.62 \\
\hline
\end{tabular}

Table S3. The relative residue propensity (RP) for each amino acid in all the proteinligand complexes containing $-\mathrm{CH}_{3}$ group.

\begin{tabular}{cccccccc}
\hline \multicolumn{2}{c}{$\begin{array}{c}\text { Intrinsic } \\
\text { Proportion (IP) }\end{array}$} & \multicolumn{3}{c}{ Occurrence Proportion (OP) } & \multicolumn{2}{c}{ Relative Residues Preferences (RP) } \\
\hline \multicolumn{2}{c}{ Freq } & $d<3 \AA$ & $3 \AA \leq d<4 \AA$ & $4 \AA \leq d<5 \AA$ & $d<3 \AA$ & $3 \AA \leq d<4 \AA$ & $4 \AA \leq d<5 \AA$ \\
Ala & 7.95 & 7.28 & 6.04 & 5.44 & 0.92 & 0.76 & 0.69 \\
Cys & 1.38 & 2.10 & 1.40 & 1.99 & 1.52 & 1.02 & 1.45 \\
Asp & 5.46 & 1.15 & 1.94 & 2.08 & 0.21 & 0.35 & 0.38 \\
Glu & 6.52 & 2.96 & 2.61 & 2.25 & 0.45 & 0.40 & 0.34 \\
Phe & 3.98 & 5.44 & 10.94 & 11.25 & 1.37 & 2.75 & 2.83 \\
Gly & 7.34 & 2.25 & 4.11 & 3.91 & 0.31 & 0.56 & 0.53 \\
His & 2.65 & 1.40 & 2.90 & 3.22 & 0.53 & 1.09 & 1.22 \\
Ile & 5.55 & 8.35 & 8.01 & 8.11 & 1.50 & 1.44 & 1.46 \\
Lys & 6.03 & 3.13 & 2.23 & 2.51 & 0.52 & 0.37 & 0.42 \\
Leu & 9.11 & 30.68 & 15.66 & 14.60 & 3.37 & 1.72 & 1.60 \\
9 & & & & & &
\end{tabular}




\begin{tabular}{llllllll} 
Met & 2.32 & 6.18 & 4.02 & 4.37 & 2.67 & 1.73 & 1.89 \\
Asn & 4.13 & 1.94 & 2.96 & 2.50 & 0.47 & 0.72 & 0.60 \\
Pro & 4.72 & 1.74 & 2.82 & 2.66 & 0.37 & 0.60 & 0.56 \\
Gln & 3.76 & 1.28 & 2.13 & 2.16 & 0.34 & 0.57 & 0.58 \\
Arg & 5.48 & 3.12 & 3.68 & 3.49 & 0.57 & 0.67 & 0.64 \\
Ser & 6.27 & 5.09 & 3.75 & 3.52 & 0.81 & 0.60 & 0.56 \\
Thr & 5.52 & 4.02 & 4.16 & 4.12 & 0.73 & 0.75 & 0.75 \\
Val & 7.01 & 6.29 & 8.14 & 8.83 & 0.90 & 1.16 & 1.26 \\
Trp & 1.36 & 1.83 & 5.19 & 5.23 & 1.35 & 3.83 & 3.85 \\
Tyr & 3.47 & 3.78 & 7.29 & 7.75 & 1.09 & 2.10 & 2.24 \\
\hline
\end{tabular}

Table S4. Ligand structures containing trifluoromethyl-benzene ring for QM/MM calculation.

\begin{tabular}{|c|c|c|c|c|}
\hline Number & $\begin{array}{c}\text { PDB- } \\
\text { id }\end{array}$ & Ligand Structure & Bioactivity & Resolution \\
\hline 1 & $3 \mathrm{~h} 7 \mathrm{w}$ & & $\begin{array}{c}\mathrm{Kd}: 590 \\
\mathrm{nM}\end{array}$ & $1.65 \AA$ \\
\hline 2 & 3 we4 & & $\mathrm{IC}_{50}: 15 \mathrm{nM}$ & $2.00 \AA$ \\
\hline 3 & 4ufu & & $\mathrm{IC}_{50}: 10 \mathrm{nM}$ & $2.10 \AA$ \\
\hline 4 & $3 n 3 g$ & & $\begin{array}{c}\mathrm{Ki} / \mathrm{IC} C_{50}: 58 \\
\mathrm{nM}\end{array}$ & $1.60 \AA$ \\
\hline 5 & $4 \mathrm{j} \times 7$ & & $\mathrm{IC}_{50}: 123$ & $2.40 \AA$ \\
\hline
\end{tabular}




\begin{tabular}{|c|c|c|c|}
\hline 6 & $3 \mathrm{~d} 14$ & $\mathrm{IC}_{50}: 22 \mathrm{nM}$ & $1.90 \AA$ \\
\hline 7 & $2 \mathrm{duz}$ & $\begin{array}{c}\mathrm{IC}_{50}: 4 \sim 81 \\
\mathrm{nM}\end{array}$ & $1.60 \AA$ \\
\hline 8 & $4 \mathrm{j} 8 \mathrm{~m}$ & $\mathrm{IC}_{50}: 48 \mathrm{nM}$ & $1.85 \AA$ \\
\hline 9 & 4ui3 & $\begin{array}{c}\mathrm{IC}_{50}: 133 \\
\mathrm{nM}\end{array}$ & $2.00 \AA$ \\
\hline 10 & $3 \mathrm{poz}$ & $\begin{array}{c}\mathrm{IC}_{50}: 1 \sim 23 \\
\mathrm{nM}\end{array}$ & $1.50 \AA$ \\
\hline 11 & $3 b g q$ & $\mathrm{Ki}: 11 \mathrm{nM}$ & $2.00 \AA$ \\
\hline 12 & $4 \mathrm{~g} 31$ & $\begin{array}{c}\mathrm{IC}_{50}: \\
0.4 \sim 30 \mathrm{nM}\end{array}$ & $2.28 \AA$ \\
\hline
\end{tabular}




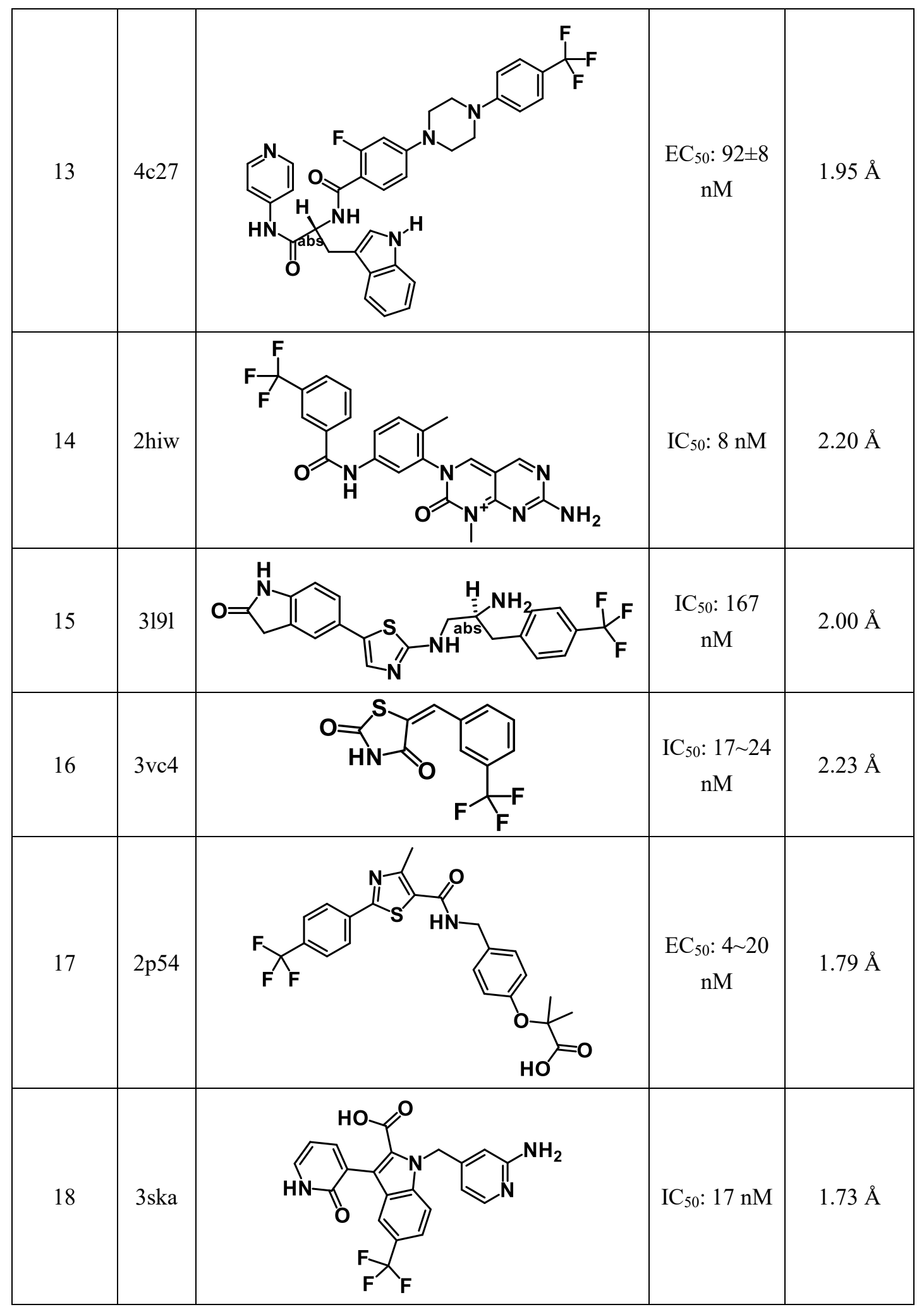




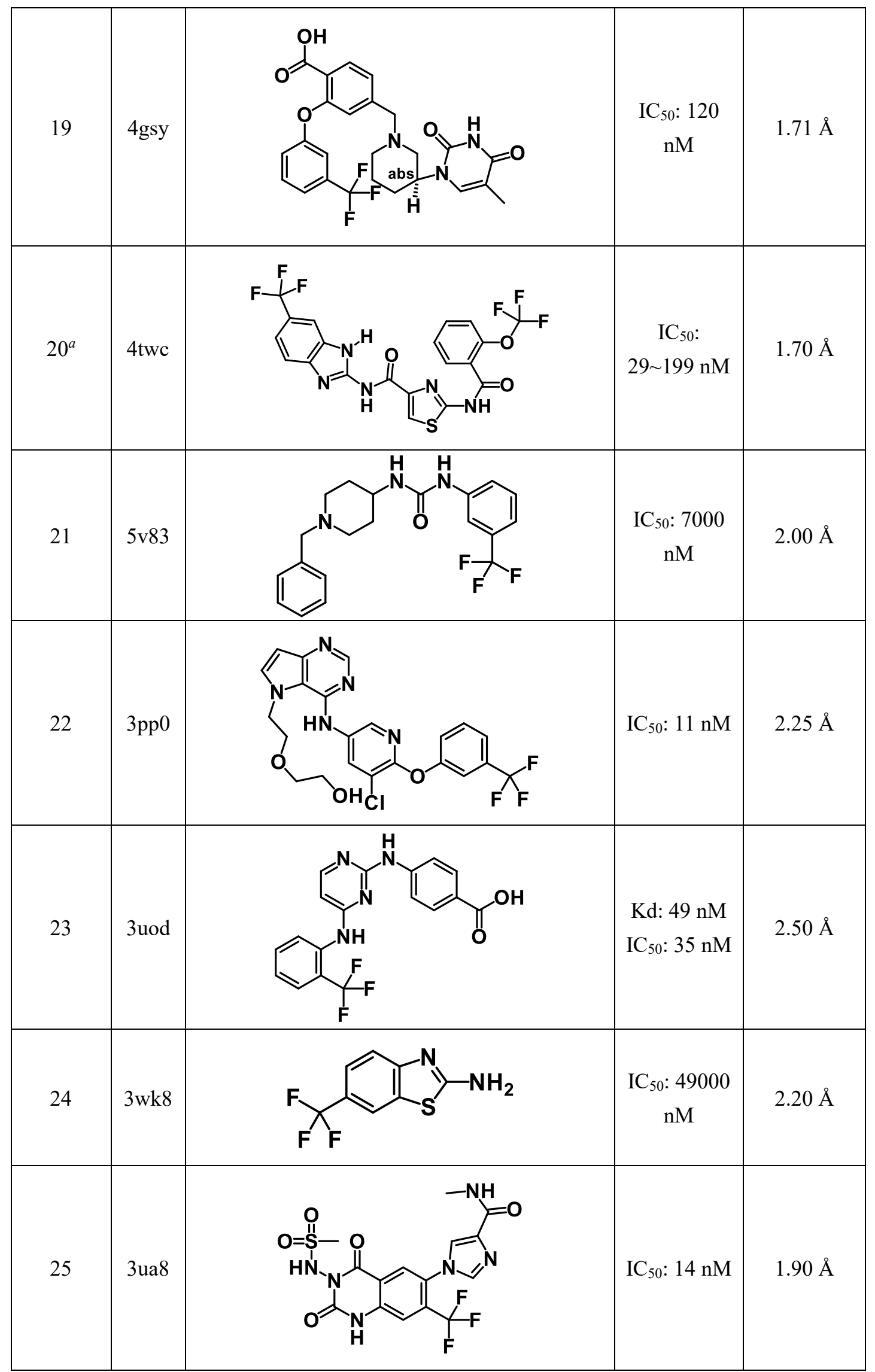




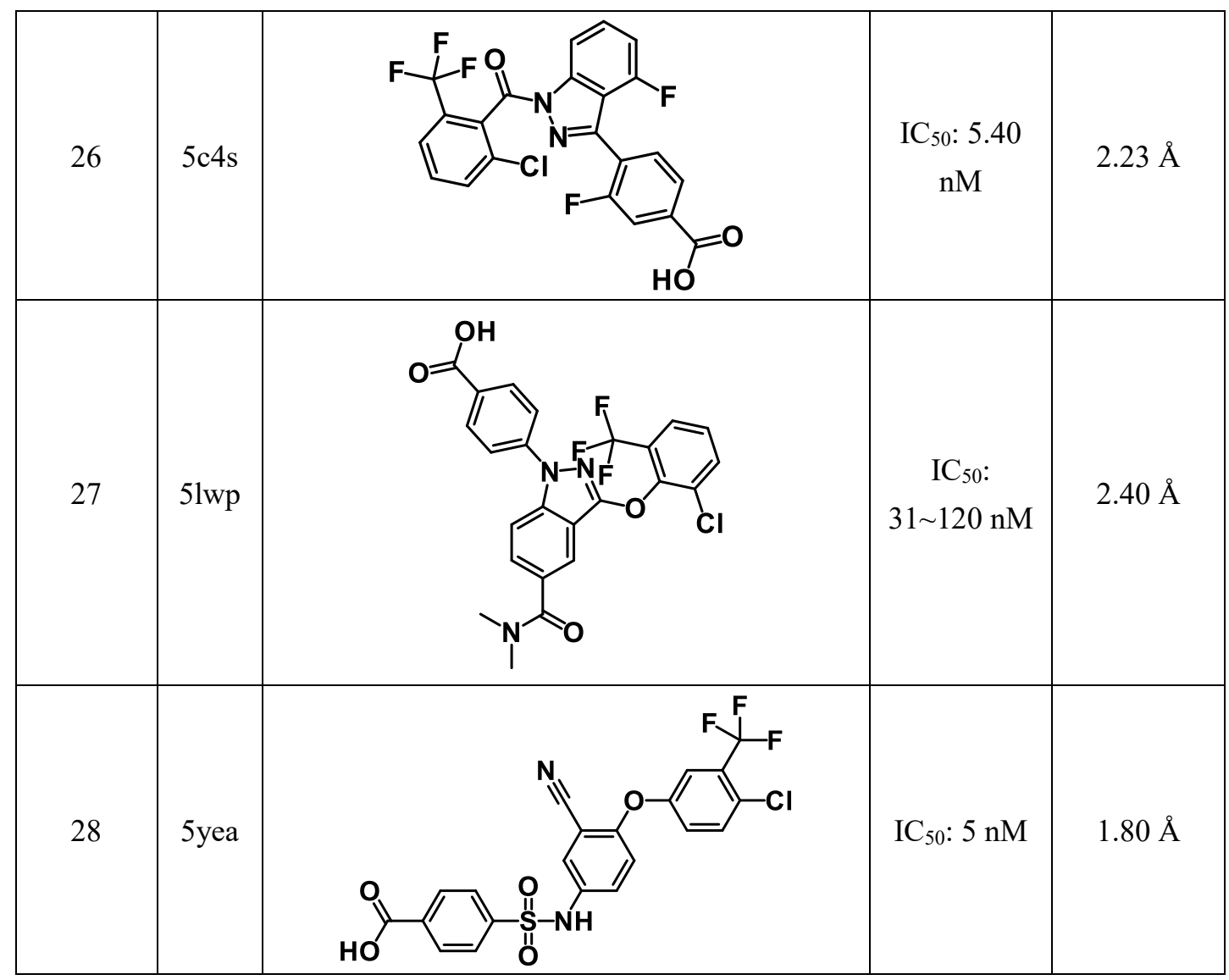

${ }^{a}$ : We only focus on the $-\mathrm{Phe}-\mathrm{CF}_{3}$ substructure in the calculations. 
Table S5. Ligand structures with trifluoromethyl not attached to benzene ring for QM/MM.

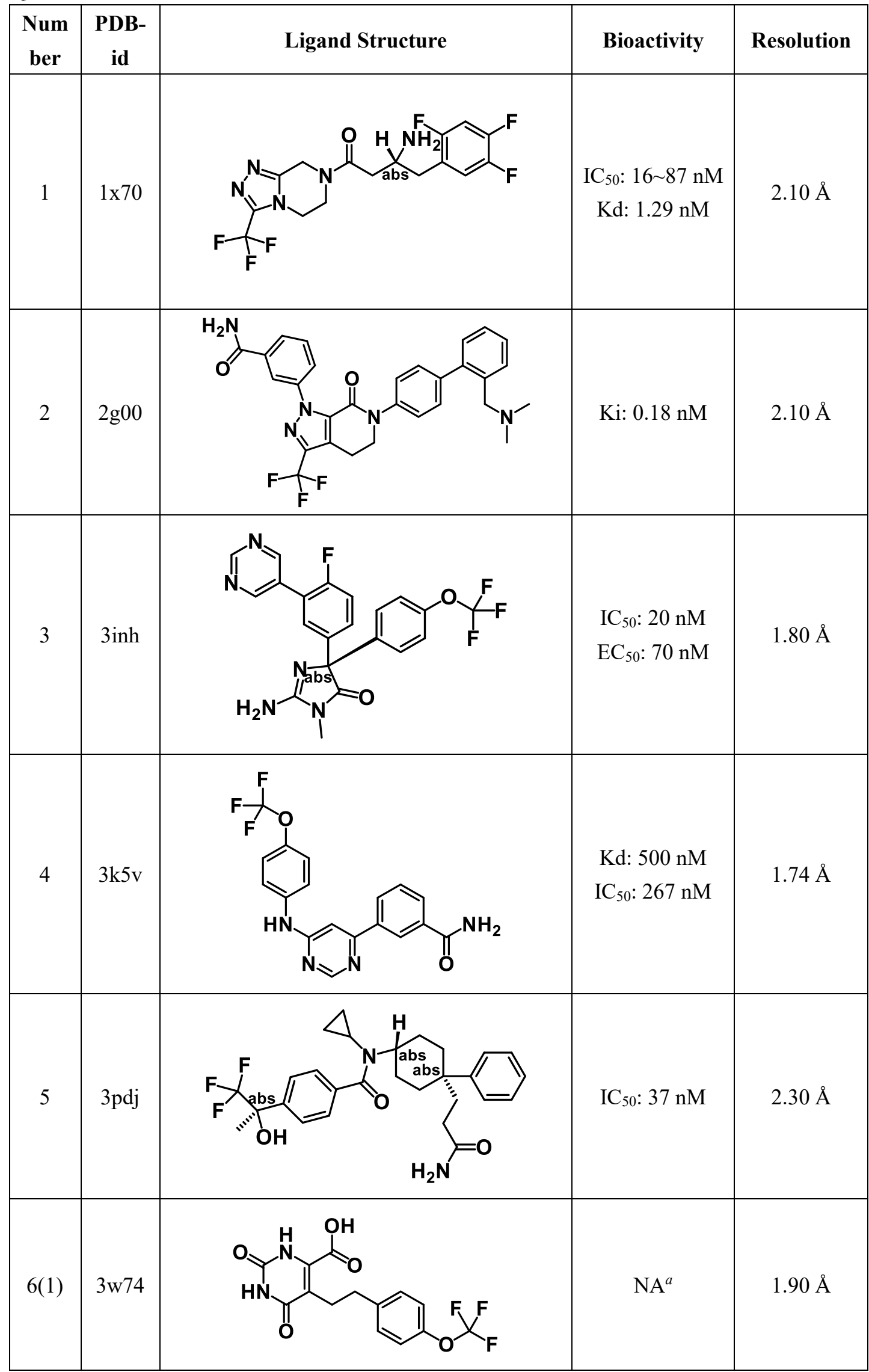




(2)

${ }^{a}$ NA: Not Available

${ }^{b}$ : No. 6(2) does not have $-\mathrm{CF}_{3}$, it forms a compound pair with No. 6(1) by only altering $-\mathrm{CF}_{3}$ to $-\mathrm{CH}_{3}$. 
Table S6 Total binding free energy ( $\left.\Delta G_{\text {Total }}\right)$ of 27 complexes pairs and ligand structures from MM/GBSA.

\begin{tabular}{|c|c|c|c|c|c|}
\hline \multirow{2}{*}{$\begin{array}{c}\text { Num } \\
\text { ber }\end{array}$} & \multirow{2}{*}{$\begin{array}{c}\text { PDB } \\
\text {-id }\end{array}$} & \multirow{2}{*}{ Ligand Structure } & \multirow{2}{*}{$\begin{array}{l}\text { Ligand } \\
\text { Charge } \\
\end{array}$} & \multicolumn{2}{|c|}{$\Delta G_{\text {Total }}(\mathrm{kcal} / \mathrm{mol})$} \\
\hline & & & & $-\mathrm{CF}_{3}$ & $-\mathrm{CH}_{3}$ \\
\hline 1 & $2 \mathrm{~g} 00$ & & 1 & $-16.87 \pm 0.61$ & $-15.11 \pm 0.70$ \\
\hline 2 & $2 \mathrm{p} 54$ & & 0 & $-47.11 \pm 0.39$ & $-40.51 \pm 0.46$ \\
\hline 3 & 2 yac & & 0 & $-23.23 \pm 0.55$ & $-21.09 \pm 0.56$ \\
\hline 4 & $4 \mathrm{jx} 7$ & & 0 & $-20.93 \pm 0.31$ & $-14.99 \pm 0.39$ \\
\hline 5 & $3 \mathrm{~d} 14$ & & 0 & $-49.53 \pm 0.71$ & $-42.38 \pm 0.80$ \\
\hline 6 & $3 p d j$ & & 0 & $-4.38 \pm 0.50$ & $-9.13 \pm 0.63$ \\
\hline
\end{tabular}




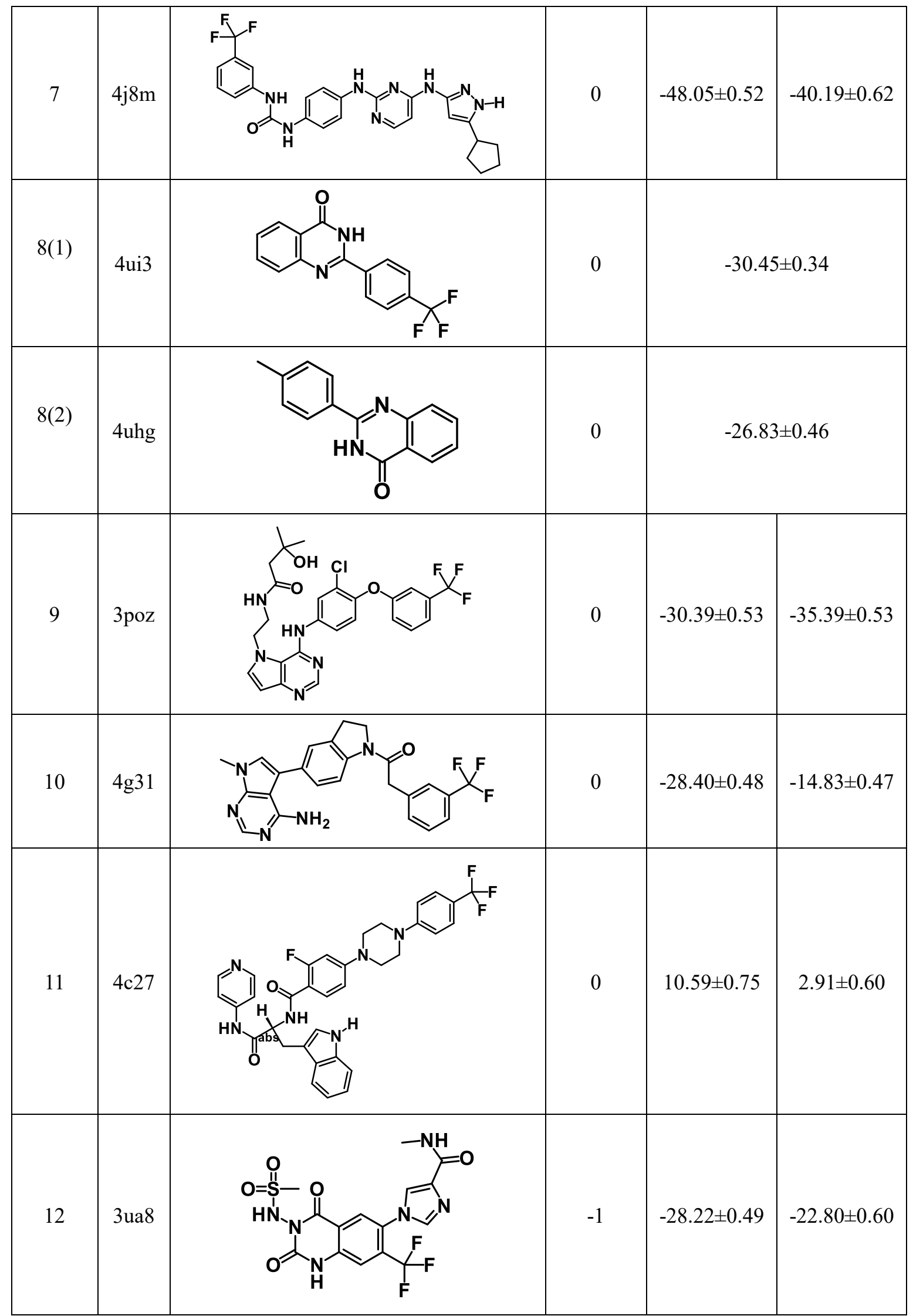




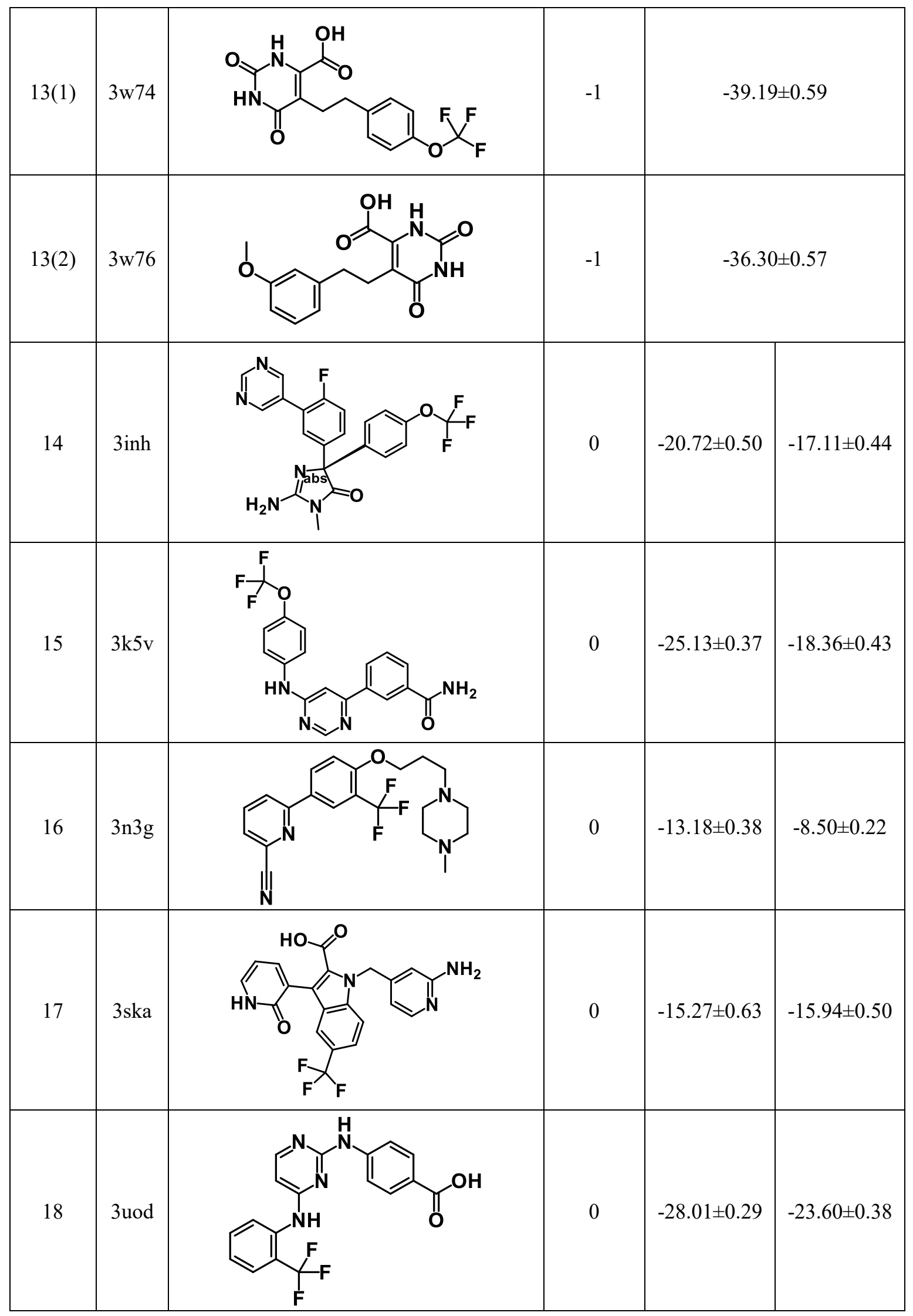




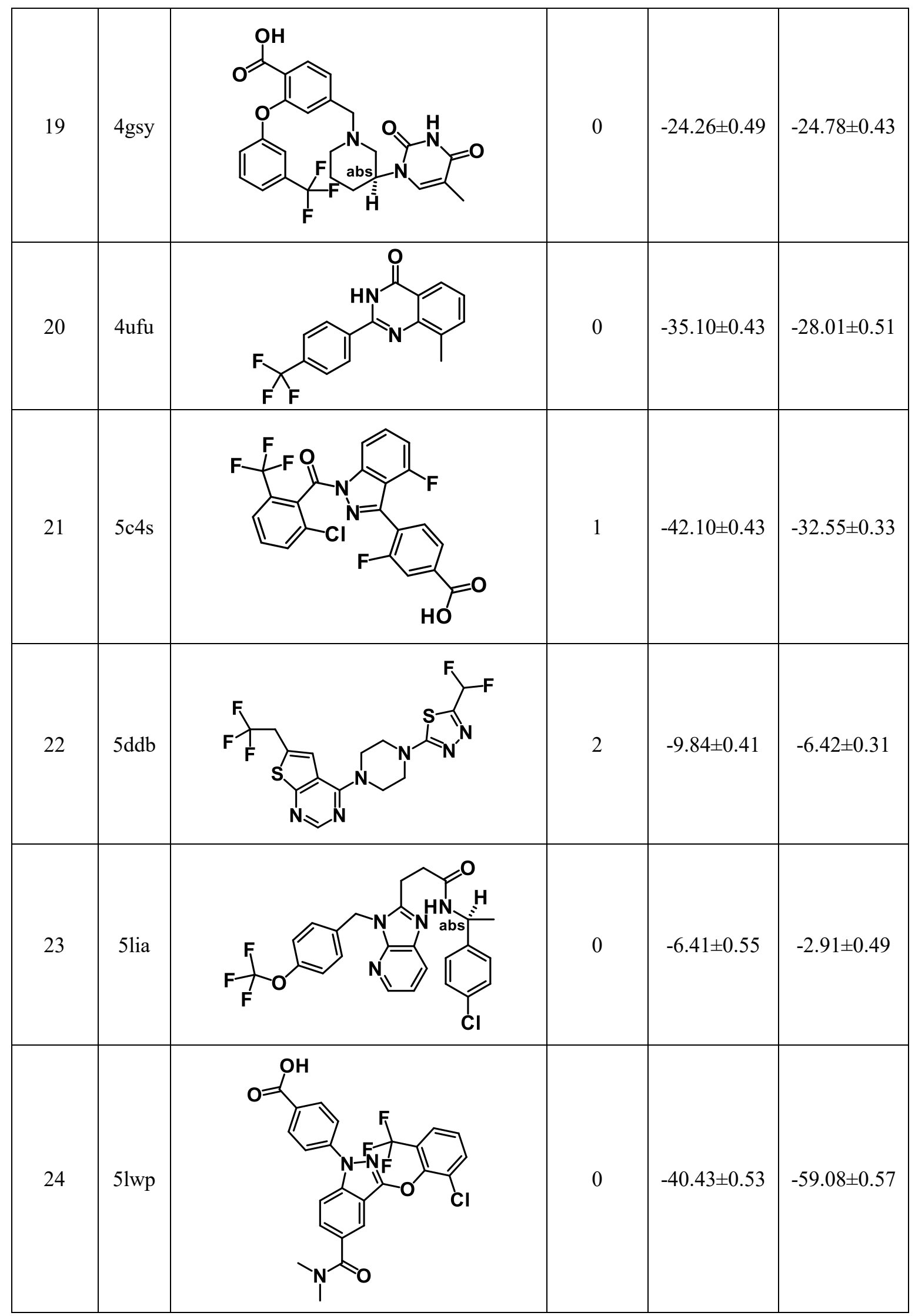




25026

Table S7 Energy decomposition by using MM/GBSA method for all $-\mathrm{CF}_{3}$ systems.

\begin{tabular}{|c|c|c|c|c|c|c|c|}
\hline $\begin{array}{l}\text { PDB- } \\
\text { id }\end{array}$ & $E_{\mathrm{vdw}}$ & $E_{\text {ele }}$ & $E_{\mathrm{gb}}$ & $E_{\text {surf }}$ & $\Delta G_{\text {TOTAL }}$ & $\begin{array}{l}\text { Binding } \\
\text { Affinity }\end{array}$ & $\begin{array}{c}\Delta G_{-} \text {exp } \\
\text { Kcal } / \text { mol }\end{array}$ \\
\hline $2 \mathrm{~g} 00$ & -53.27 & -40.62 & 82.96 & -5.94 & -16.87 & $\mathrm{~K}_{\mathrm{i}}: 0.18 \mathrm{nM}$ & -13.31 \\
\hline $2 \mathrm{p} 54$ & -61.64 & -23.06 & 46.33 & -8.74 & -47.11 & $\mathrm{EC}_{50}: 20 \mathrm{nM}$ & -10.51 \\
\hline 2 yac & -58.08 & -48.29 & 90.32 & -7.18 & -23.23 & $\mathrm{IC}_{50}: 2 \mathrm{nM}$ & -11.88 \\
\hline $3 \mathrm{~d} 14$ & -55.36 & -109.72 & 123.45 & -7.91 & -49.53 & $\mathrm{IC}_{50}: 22 \mathrm{nM}$ & -10.46 \\
\hline $3 p d j$ & -45.75 & -26.78 & 74.62 & -6.48 & -4.38 & $\mathrm{EC}_{50}: 0.8 \mathrm{nM}$ & -12.42 \\
\hline 3 ua8 & -30.88 & -145.01 & 152.65 & -4.98 & -28.22 & $\mathrm{IC}_{50}: 14 \mathrm{nM}$ & $\begin{array}{r}-10.72 \\
-9.37\end{array}$ \\
\hline $3 w 74$ & -21.54 & -113.01 & 99.48 & -4.12 & -39.19 & ${ }^{a} \mathrm{NA}$ & \\
\hline 3 inh & -42.11 & -69.57 & 97.44 & -6.47 & -20.72 & $\mathrm{IC}_{50}: 40 \mathrm{nM}$ & -10.10 \\
\hline $3 \mathrm{k} 5 \mathrm{v}$ & -41.31 & -31.79 & 53.37 & -5.41 & -25.13 & $\mathrm{IC}_{50}: 267 \mathrm{nM}$ & -8.98 \\
\hline
\end{tabular}




\begin{tabular}{|c|c|c|c|c|c|c|c|}
\hline $3 n 3 g$ & -27.72 & -5.46 & 23.58 & -3.58 & -13.18 & $\mathrm{IC}_{50}: 5 \mathrm{nM}$ & -11.33 \\
\hline $3 \mathrm{poz}$ & -70.91 & -49.05 & 98.19 & -8.59 & -30.36 & $\mathrm{IC}_{50}: 1 \mathrm{nM}$ & -12.29 \\
\hline 3 ska & -39.02 & -37.53 & 66.71 & -5.43 & -15.27 & $\mathrm{IC}_{50}: 17 \mathrm{nM}$ & -10.61 \\
\hline 3 uod & -40.92 & -34.13 & 52.40 & -5.36 & -28.01 & $\mathrm{IC}_{50}: 49 \mathrm{nM}$ & -9.98 \\
\hline $4 c 27$ & -68.48 & -18.54 & 106.55 & -8.94 & 10.59 & $\mathrm{IC}_{50}: 84 \mathrm{nM}$ & -9.66 \\
\hline $4 \mathrm{~g} 31$ & -61.07 & -24.55 & 64.63 & -7.41 & -28.40 & $\mathrm{IC}_{50}: 0.4 \mathrm{nM}$ & -12.83 \\
\hline 4gsy & -47.71 & -115.79 & 145.49 & -6.25 & -24.26 & $\mathrm{IC}_{50}: 120 \mathrm{nM}$ & -9.45 \\
\hline 4ufu & -41.68 & -33.77 & 45.27 & -4.92 & -35.10 & $\mathrm{IC}_{50}: 10 \mathrm{nM}$ & -10.92 \\
\hline $4 \mathrm{j} 8 \mathrm{~m}$ & -57.44 & -123.95 & 141.66 & -8.32 & -48.05 & $\mathrm{IC}_{50}: 48 \mathrm{nM}$ & -9.99 \\
\hline $4 \mathrm{j} \times 7$ & -45.47 & -22.15 & 52.74 & -6.04 & -20.93 & $\mathrm{IC}_{50}: 123 \mathrm{nM}$ & -9.44 \\
\hline $4 \mathrm{twc}$ & -44.87 & -57.57 & 79.61 & -5.90 & -28.73 & $\mathrm{IC}_{50}: 40 \mathrm{nM}$ & -10.10 \\
\hline $5 \mathrm{c} 4 \mathrm{~s}$ & -55.47 & -30.68 & 51.64 & -7.58 & -42.10 & $\mathrm{IC}_{50}: 5.4 \mathrm{nM}$ & -11.29 \\
\hline $5 \mathrm{ddb}$ & -39.74 & -36.55 & 71.39 & -4.93 & -9.84 & $\mathrm{IC}_{50}: 82 \mathrm{nM}$ & -9.68 \\
\hline 5lia & -63.48 & -27.67 & 92.44 & -7.68 & -6.41 & $\mathrm{EC}_{50}: 25 \mathrm{nM}$ & -10.38 \\
\hline 5lwp & -51.04 & -54.40 & 71.95 & -6.95 & -40.43 & $\begin{array}{c}\mathrm{IC}_{50}: 31 \sim 120 \\
\mathrm{nM}\end{array}$ & -10.24 \\
\hline $5 \mathrm{xkm}$ & -47.14 & -28.10 & 66.69 & -6.17 & -14.71 & $\mathrm{IC}_{50}: 24 \mathrm{nM}$ & -10.40 \\
\hline 5yea & -45.04 & -58.82 & 95.38 & -6.57 & -15.06 & $\mathrm{IC}_{50}: 5 \mathrm{nM}$ & -11.33 \\
\hline 4ui3 & -39.44 & -19.52 & 33.16 & -4.64 & -30.45 & $\mathrm{IC}_{50}: 133 \mathrm{nM}$ & -9.39 \\
\hline
\end{tabular}

${ }^{a}$ NA: Not Available 
Table S8 Energy decomposition by using MM/GBSA method for all $-\mathrm{CH}_{3}$ systems.

\begin{tabular}{|c|c|c|c|c|c|}
\hline PDB-id & $E_{\mathrm{vdw}}$ & $E_{\mathrm{el}}$ & $E_{\mathrm{gb}}$ & $E_{\text {surf }}$ & $\Delta \boldsymbol{G}_{\mathrm{TOTAL}}$ \\
\hline $2 \mathrm{~g} 00$ & -53.63 & -38.05 & 82.70 & -6.15 & -15.11 \\
\hline $2 \mathrm{p} 54$ & -59.48 & -30.02 & 57.00 & -8.01 & -40.51 \\
\hline 2 yac & -60.26 & -49.38 & 95.65 & -7.10 & -21.09 \\
\hline $3 \mathrm{~d} 14$ & -50.46 & -103.69 & 119.22 & -7.38 & -42.38 \\
\hline $3 \mathrm{pdj}$ & -47.98 & -38.80 & 84.28 & -6.63 & -9.13 \\
\hline 3 ua 8 & -32.96 & -123.95 & 138.88 & -4.77 & -22.80 \\
\hline $3 w 76$ & -21.57 & -111.41 & 100.71 & -4.02 & -36.30 \\
\hline $3 \mathrm{inh}$ & -40.92 & -70.57 & 100.21 & -6.47 & -17.11 \\
\hline $3 \mathrm{k} 5 \mathrm{v}$ & -41.48 & -37.48 & 65.92 & -5.32 & -18.36 \\
\hline $3 n 3 g$ & -16.68 & -10.54 & 20.90 & -2.18 & -8.50 \\
\hline $3 \mathrm{poz}$ & -69.98 & -58.70 & 101.72 & -8.43 & -35.39 \\
\hline 3 ska & -45.94 & -17.71 & 52.94 & -5.22 & -15.94 \\
\hline 3uod & -40.71 & -43.06 & 65.33 & -5.16 & -23.60 \\
\hline $4 c 27$ & -68.82 & -38.33 & 118.82 & -8.76 & 2.91 \\
\hline $4 \mathrm{~g} 31$ & -53.26 & -3.15 & 48.19 & -6.62 & -14.83 \\
\hline 4gsy & -40.87 & -48.84 & 70.51 & -5.58 & -24.78 \\
\hline 4ufu & -40.55 & -20.42 & 37.35 & -4.40 & -28.01 \\
\hline $4 \mathrm{j} 8 \mathrm{~m}$ & -55.19 & -89.71 & 112.42 & -7.71 & -40.19 \\
\hline $4 \mathrm{j} \times 7$ & -44.21 & -28.07 & 62.92 & -5.63 & -14.99 \\
\hline 4 twc & -46.07 & -46.08 & 74.82 & -5.58 & -22.91 \\
\hline $5 \mathrm{c} 4 \mathrm{~s}$ & -54.23 & -24.65 & 53.33 & -7.01 & -32.55 \\
\hline $5 \mathrm{ddb}$ & -39.17 & -27.81 & 65.11 & -4.56 & -6.42 \\
\hline 5lia & -53.28 & -18.52 & 75.61 & -6.73 & -2.91 \\
\hline 5lwp & -63.83 & -67.33 & 79.81 & -7.73 & -59.08 \\
\hline $5 \mathrm{xkm}$ & -41.70 & -39.35 & 75.84 & -5.65 & -10.86 \\
\hline 5 yea & -45.95 & -58.74 & 96.58 & -6.45 & -14.56 \\
\hline 4uhg & -40.23 & -22.91 & 40.76 & -4.45 & -26.83 \\
\hline
\end{tabular}


Table S9 Binding free energy differences and its components by using MMGBSA. $\Delta \Delta G=\Delta G_{-\mathrm{CF} 3}-\Delta G_{-\mathrm{CH} 3}(\mathrm{kcal} / \mathrm{mol})$

\begin{tabular}{|c|c|c|c|c|c|c|}
\hline Number & PDB-id & $\Delta \Delta G$ & $\Delta \Delta E_{\mathrm{vdw}}$ & $\Delta \Delta E_{\mathrm{el}}$ & $\Delta \Delta E_{\mathrm{gb}}$ & $\Delta \Delta E_{\text {surf }}$ \\
\hline 1 & $2 \mathrm{~g} 00$ & -1.76 & 0.36 & -2.57 & 0.26 & 0.21 \\
\hline 2 & $2 \mathrm{p} 54$ & -6.60 & -2.16 & 6.96 & -10.67 & -0.73 \\
\hline 3 & 2 yac & -2.14 & 2.18 & 1.09 & -5.33 & -0.08 \\
\hline 4 & $3 \mathrm{~d} 14$ & -7.15 & -4.90 & -6.03 & 4.23 & -0.53 \\
\hline 5 & $3 p d j$ & 4.75 & 2.23 & 12.02 & -9.66 & 0.15 \\
\hline 6 & 3 ua8 & -5.42 & 2.08 & -21.06 & 13.77 & -0.21 \\
\hline 7 & 3 w74/3w76 & -2.89 & 0.03 & -1.60 & -1.23 & -0.10 \\
\hline 8 & $3 \mathrm{inh}$ & -3.61 & -1.19 & 1.00 & -2.77 & 0.00 \\
\hline 9 & $3 \mathrm{k} 5 \mathrm{v}$ & -6.77 & 0.17 & 5.69 & -12.55 & -0.09 \\
\hline 10 & $3 n 3 g$ & -4.68 & -11.04 & 5.08 & 2.68 & -1.40 \\
\hline 11 & $3 \mathrm{poz}$ & 5.03 & -0.93 & 9.65 & -3.53 & -0.16 \\
\hline 12 & 3 ska & 0.67 & 6.92 & -19.82 & 13.77 & -0.21 \\
\hline 13 & 3uod & -4.41 & -0.21 & 8.93 & -12.93 & -0.20 \\
\hline 14 & $4 c 27$ & 7.68 & 0.34 & 19.79 & -12.27 & -0.18 \\
\hline 15 & $4 \mathrm{~g} 31$ & -13.57 & -7.81 & -21.40 & 16.44 & -0.79 \\
\hline 16 & 4gsy & 0.52 & -6.84 & -66.95 & 74.98 & -0.67 \\
\hline 17 & 4ufu & -7.09 & -1.13 & -13.35 & 7.92 & -0.52 \\
\hline 18 & $4 \mathrm{j} 8 \mathrm{~m}$ & -7.86 & -2.25 & -34.24 & 29.24 & -0.61 \\
\hline 19 & $4 \mathrm{j} \times 7$ & -5.94 & -1.26 & 5.92 & -10.18 & -0.41 \\
\hline 20 & 4 twc & -5.82 & 1.20 & -11.49 & 4.80 & -0.32 \\
\hline 21 & $5 \mathrm{c} 4 \mathrm{~s}$ & -9.55 & -1.24 & -6.03 & -1.69 & -0.57 \\
\hline 22 & $5 \mathrm{ddb}$ & -3.42 & -0.57 & -8.74 & 6.28 & -0.37 \\
\hline 23 & 5lia & -3.50 & -10.20 & -9.15 & 16.83 & -0.95 \\
\hline 24 & 5lwp & 18.65 & 12.79 & 12.93 & -7.86 & 0.78 \\
\hline 25 & $5 \mathrm{xkm}$ & -3.85 & -5.44 & 11.25 & -9.15 & -0.52 \\
\hline 26 & 5 yea & -0.50 & 0.91 & -0.08 & -1.20 & -0.12 \\
\hline 27 & 4ui3/4uhg & -3.62 & 0.79 & 3.39 & -7.60 & -0.19 \\
\hline
\end{tabular}


Table S10 Energy Differences $\left(\Delta \Delta E=\Delta E_{-\mathrm{CF} 3}-\Delta E_{-\mathrm{CH} 3}\right)(\mathrm{kcal} / \mathrm{mol})$ from QM/MM calculation in vacuum, TCM, and DCE, respectively.

\begin{tabular}{|c|c|c|c|}
\hline PDB-id & $\Delta \Delta E_{\text {Vacuum }}$ & $\Delta \Delta E_{\mathrm{TCM}}$ & $\Delta \Delta E_{\mathrm{DCE}}$ \\
\hline $3 \mathrm{~h} 7 \mathrm{w}$ & -4.98 & -4.36 & -4.28 \\
\hline 3we4 & -4.89 & -3.50 & -3.92 \\
\hline 4ufu & -1.73 & -1.46 & -1.40 \\
\hline $3 n 3 g$ & -1.70 & -1.06 & -0.81 \\
\hline $4 \mathrm{j} \times 7$ & -1.64 & -1.09 & -0.87 \\
\hline $3 \mathrm{~d} 14$ & -1.57 & -0.73 & -0.48 \\
\hline 2duz & -1.52 & -0.24 & 0.03 \\
\hline $4 \mathrm{j} 8 \mathrm{~m}$ & -0.90 & -0.57 & -0.14 \\
\hline 4ui3/4uhg & -0.70 & -0.48 & -0.46 \\
\hline $3 \mathrm{poz}$ & -0.56 & -0.28 & -0.18 \\
\hline $3 b g q$ & -0.44 & 0.03 & 0.19 \\
\hline $4 \mathrm{~g} 31$ & -0.29 & 0.11 & 0.16 \\
\hline $4 c 27$ & -0.20 & 0.07 & 0.11 \\
\hline 2hiw & -0.09 & 0.00 & 0.00 \\
\hline 3191 & -0.06 & 0.01 & 0.26 \\
\hline $3 \mathrm{vc} 4$ & -0.05 & 0.14 & 0.16 \\
\hline $2 \mathrm{p} 54$ & -0.03 & 0.00 & 0.00 \\
\hline 3 ska & 0.01 & 0.40 & 0.47 \\
\hline 4gsy & 0.08 & 0.30 & 0.31 \\
\hline 4 twc & 0.12 & 0.55 & 0.57 \\
\hline $5 v 83$ & 0.37 & 0.52 & 0.60 \\
\hline $3 p p 0$ & 0.85 & 1.04 & 1.02 \\
\hline 3uod & 5.05 & 1.81 & 1.15 \\
\hline $1 \times 70$ & -2.02 & -2.26 & -2.02 \\
\hline $2 \mathrm{~g} 00$ & -0.40 & -0.21 & -0.15 \\
\hline 3 inh & -0.50 & -0.21 & -0.16 \\
\hline $3 \mathrm{k} 5 \mathrm{v}$ & 0.79 & 0.72 & 0.64 \\
\hline $3 \mathrm{pdj}$ & 0.39 & 0.23 & 0.10 \\
\hline 3 ua8 & 1.92 & 1.02 & 0.86 \\
\hline $3 w 74 / 3 w 76$ & 0.37 & 0.40 & 0.13 \\
\hline $3 \mathrm{wk} 8$ & 1.86 & 1.28 & 1.08 \\
\hline $5 \mathrm{c} 4 \mathrm{~s}$ & 0.12 & 0.29 & 0.31 \\
\hline $5 \mathrm{ddb}$ & -0.33 & -0.26 & -0.25 \\
\hline $5 \mathrm{jwc}$ & -1.07 & -1.17 & -1.21 \\
\hline 5lia & -2.66 & -2.55 & -2.50 \\
\hline 5lwp & 0.71 & 0.47 & 0.40 \\
\hline 5 nud & 1.75 & 1.60 & -0.32 \\
\hline $5 \times \mathrm{km}$ & 0.05 & 0.23 & 0.26 \\
\hline 5yea & 0.55 & 0.60 & 0.60 \\
\hline
\end{tabular}


Table S11 Average of binding free energy decomposition on amino acids by using $\operatorname{MMGBSA}(\mathrm{kcal} / \mathrm{mol})$.

\begin{tabular}{ccccc}
\hline Amino acids & Mean of $-\mathbf{C F}_{\mathbf{3}}$ & Mean of $-\mathbf{C H}_{\mathbf{3}}$ & Stdev of $-\mathbf{C F}_{\mathbf{3}}$ & Stdev of $-\mathbf{C H}_{\mathbf{3}}$ \\
\hline Arg & -3.38 & -3.12 & 0.18 & -0.26 \\
Met & -3.35 & -2.42 & 0.66 & -0.93 \\
Phe & -2.80 & -1.75 & 0.74 & -1.04 \\
Trp & -2.50 & -2.03 & 0.33 & -0.47 \\
Tyr & -2.22 & -2.15 & 0.05 & -0.07 \\
Asn & -2.14 & -1.62 & 0.37 & -0.52 \\
Asp & -2.11 & -1.30 & 0.57 & -0.81 \\
Gln & -2.07 & -2.04 & 0.02 & -0.03 \\
Val & -1.50 & -1.38 & 0.08 & -0.12 \\
Glu & -3.59 & -4.41 & 0.58 & 0.82 \\
Gly & -2.59 & -3.05 & 0.33 & 0.46 \\
Cys & -2.39 & -2.62 & 0.16 & 0.23 \\
His & -2.37 & -2.61 & 0.17 & 0.24 \\
Lys & -2.15 & -3.42 & 0.90 & 1.27 \\
Leu & -2.03 & -2.37 & 0.23 & 0.33 \\
Thr & -2.03 & -2.05 & 0.02 & 0.03 \\
Ser & -1.97 & -1.98 & 0.01 & 0.01 \\
Ala & -1.94 & -1.97 & 0.02 & 0.03 \\
Ile & -1.81 & -1.92 & 0.08 & 0.11 \\
Pro & -1.76 & -1.97 & 0.15 & 0.21 \\
\hline
\end{tabular}

\section{References}

1. Lu, T.; Chen, F., Multiwfn: a multifunctional wavefunction analyzer. J. Comput. Chem. 2012, 33 (5), 580-592. 